\title{
ABSTRACT INCOMPLETE DEGENERATE DIFFERENTIAL EQUATIONS
}

\author{
By \\ Marko Kostić
}

\begin{abstract}
In this paper, we investigate the abstract incomplete degenerate differential equations in locally convex spaces, associated with use of the modified Liuoville right-sided fractional derivatives ([21]). The existence of solutions of abstract incomplete degenerate differential equations of second order is proved by considering the corresponding incomplete degenerate differential equations of order $1 / \gamma \quad(0<\gamma<1 / 2)$ and using an approximation process when $\gamma \rightarrow 1 / 2-$.
\end{abstract}

\section{Introduction and Preliminaries}

The theory of abstract degenerate differential equations is still an active field of research (cf. [2], [5], [10], [12]-[16], [33]-[35], [40]-[41] and [43]-[45] for the basic source of information). To the best of our knowledge, almost nothing has been said about the abstract incomplete degenerate differential equations so far. That was a strong motivational factor that influenced us to write this paper.

The organization and main ideas of paper can be briefly described as follows. It is well known that semigroups of growth order $r>0$ were introduced by G. Da Prato [8] in 1966 (we refer the reader to [20, Section 1.2, Section 1.4] and references cited there for more details concerning this topic); the class of $C$-regularized semigroups of growth order $r>0$ has been recently introduced in

2010 Mathematics Subject Classification: 47D06, 47D60, 47D62, 47D99.

Key words and phrases: abstract incomplete differential equations, abstract degenerate differential equations, modified Liuoville right-sided time-fractional derivatives, approximations, degenerate $(B, C)$-regularized semigroups of growth order $r>0$, abstract differential operators.

The author is partially supported by grant 174024 of Ministry of Science and Technological Development, Republic of Serbia.

Received July 1, 2015.

Revised June 13, 2016. 
[6]. For the purpose of research of abstract degenerate incomplete Cauchy problems, in this paper we introduce the concept of degenerate $(B, C)$-regularized semigroups of growth order $r>0$ and clarify their most important structural properties (the full analysis of introduced class is very non-trivial and falls out from the framework of this paper; cf. Definition 2.1-Definition 2.2, Remark 2.3 and the paragraph following it). Our main results are Theorem 2.5 and Theorem 2.6. In Theorem 2.5, we consider the following abstract incomplete degenerate differential equations with modified Liouville right-sided fractional derivatives:

$$
\left(F P_{\alpha_{1}, \beta_{1}, \theta}\right):\left\{\begin{array}{l}
u \in C^{\infty}((0, \infty): E) \\
D_{-}^{\alpha_{1}} B D_{-}^{\beta_{1}} u(s)=e^{i \theta / \gamma} A u(s), \quad s>0 \\
\lim _{s \rightarrow 0+} B u(s)=C x \\
\text { the set }\{B u(s): s>0\} \text { is bounded in } E
\end{array}\right.
$$

and

$$
\left(F P_{\alpha_{1}, \beta_{1}, \theta}\right)^{\prime}:\left\{\begin{array}{l}
u \in C^{\infty}((0, \infty): E) \\
D_{-}^{\alpha_{1}} B D_{-}^{\beta_{1}} u(s)=e^{i \theta / \gamma} A u(s), \quad s>0 \\
\lim _{s \rightarrow 0+} B u(s)=C x \\
\text { the sets }\left\{\left(1+s^{-(q+1) / \gamma}\right)^{-1} u(s): s>0\right\} \\
\text { and }\left\{\left(1+s^{-(q+1) / \gamma}\right)^{-1} B u(s): s>0\right\} \text { are bounded in } E
\end{array}\right.
$$

here, $E$ is a Hausdorff sequentially complete locally convex space over the field of complex numbers (the state space), $0<\gamma<1 / 2, \alpha_{1} \geq 0, \beta_{1} \geq 0, \alpha_{1}+\beta_{1}=1 / \gamma$, $\theta \in(\gamma \pi-(\pi / 2),(\pi / 2)-\gamma \pi), A$ and $B$ are closed linear operators acting on $E, C \in L(E)$ is injective, $A$ belongs to the class $\mathscr{M}_{B, C, q}$ introduced below, and $q>-1$, resp. $-1-\gamma<q \leq-1$, in the case of consideration of problem $\left(F P_{\alpha_{1}, \beta_{1}, \theta}\right)$, resp. $\left(F P_{\alpha_{1}, \beta_{1}, \theta}\right)^{\prime}$. On the other hand, in Theorem 2.6 we consider the following abstract incomplete degenerate Cauchy problem of second order

$$
\left(P_{2, q, B}\right):\left\{\begin{array}{l}
u \in \mathscr{A}\left(\Sigma_{(\pi / 2)-(\omega / 2)}: E\right), \quad B u \in \mathscr{A}\left(\Sigma_{(\pi / 2)-(\omega / 2)}: E\right) \\
B u^{\prime \prime}(z)=\frac{d^{2}}{d z^{2}} B u(z)=A u(z), \quad z \in \Sigma_{(\pi / 2)-(\omega / 2)} \\
\lim _{z \rightarrow 0, z \in \Sigma_{\delta}} B u(z)=C x, \quad \text { for every } \delta \in(0,(\pi / 2)-(\omega / 2)), \\
\text { the sets }\left\{\left(1+|z|^{-(2 q+2)}\right)^{-1} u(z): z \in \Sigma_{\delta}\right\} \text { and } \\
\left\{\left(1+|z|^{-(2 q+2)}\right)^{-1} B u(z): z \in \Sigma_{\delta}\right\} \text { are bounded in } E \\
\text { for every } \delta \in(0,(\pi / 2)-(\omega / 2)),
\end{array}\right.
$$

where $(-3) / 2<q<(-1) / 2, \mathscr{A}\left(\Sigma_{(\pi / 2)-(\omega / 2)}: E\right)$ denotes the set consisting of all analytic functions from the sector $\Sigma_{(\pi / 2)-(\omega / 2)}=\{\lambda \in \mathbf{C} \backslash\{0\}:|\arg (\lambda)|<(\pi / 2)-$ 
$(\omega / 2)\}$ into $E$, and the condition $(\mathrm{H})$ stated below holds with some number $\omega \in[0, \pi)$. In doing so, we factically continue our previous researches raised in [6]-[7] (cf. [21, Section 2.9, Addendum] for a detailed exposition of results, and some mistakes made in previous investigations), and contribute to the theory of abstract degenerate fractional differential equations, which is in its very early stage (cf. [17]-[18] and [23]-[29] for some recent results in this direction).

The proof of Theorem 2.5 leans heavily on our results from Theorem 2.4, in which we analyze the qualitative properties of operator family $\left(W_{\gamma}(t)\right)_{t>0}$ $(\gamma \in(0,1 / 2))$ following the ideas of A. V. Balakrishnan [3] and C. Martinez, M. Sanz, A. Redondo [31]. In contrast to the assertions of Theorem 2.4-Theorem 2.5 , whose proofs can be regarded, more or less, as a technical modification of the proof of [21, Theorem 2.9.48], the proof of Theorem 2.6 is essentially different from that of [21, Theorem 2.9.51(iii)], where a non-degenerate analogon has been considered. Speaking-matter-of-factly, the method used in the proof of last mentioned theorem is based on the argumentation used in that of [3, Theorem 5.1] (cf. also [30, Theorem 5.5.2]), which does not work any longer in degenerate case for proving the fact that the function $z \mapsto W_{1 / 2}(z) x$ is a solution of the problem $\left(P_{2, q, B}\right)$ (cf. the formulation and proof of Theorem 2.6 for more details). To see this, we apply Theorem 2.5 and the approximation process when $\gamma \rightarrow 1 / 2-$.

It is clear that Theorem 2.5 and Theorem 2.6 provide generalizations of some assertions from [21, Section 2.9]: we feel duty bound to say that some of these generalizations are complete and some of them are only partial. For example, our results on the abstract degenerate Cauchy problem $\left(F P_{\alpha_{1}, \beta_{1}, \theta}\right)$ basically strengthens the corresponding results on the abstract non-degenerate Cauchy problem $\left(F P_{\beta}\right)$ (cf. [21, Theorem 2.9.48(ix.1)]) but we cannot conclude, as in the formulation of this theorem, that the corresponding solution $t \mapsto u(t), t>0$ of problem $\left(F P_{\alpha_{1}, \beta_{1}, \theta}\right)$ satisfies the property that for each $n \in \mathbf{N}$ the mapping $t \mapsto A^{n} u(t), \quad t>0$ is well-defined and continuous. On the other hand, the abstract Cauchy problem $\left(F P_{\beta, m}\right)$, appearing in the formulation of [21, Theorem 2.9.48(ix.2)], cannot be so easily considered in degenerate case because we do not know what would be the fractional power $(A+\epsilon)_{\gamma \beta}$ in the newly arisen situation (cf. also the problems $\left(F P_{\alpha_{1}, \beta_{1}, \theta}\right)$ and $\left(F P_{\alpha_{1}, \beta_{1}, \theta}\right)^{\prime}$, in which we work with the operator $A$, not with its fractional power $A_{\beta \gamma}$, as in [21, Theorem 2.9.48(ix.1)]). In the case of consideration of abstract incomplete degenerate Cauchy problems of second order, we are losing some valuable information, as well (see e.g. the inclusion $D\left(A_{1 / 2}\right) \cup D(A) \subseteq \Omega_{1 / 2}$, appearing in the formulation of [21, Theorem 2.9.51(iii.1)]). We apply Theorem 2.5 and Theorem 2.6 in the analysis of some 
incomplete degenerate Cauchy problems associated with abstract differential operators ([21], [46]). It should be finally observed that the furnished applications as well as our obtained theoretical results on the well-posedness of problems $\left(F P_{\alpha_{1}, \beta_{1}, \theta}\right),\left(F P_{\alpha_{1}, \beta_{1}, \theta}\right)^{\prime}$ and $\left(P_{2, q, B}\right)$ seem to be valuable only from the mathematical point of view and do not have an important physical significance, for now at least.

We use the standard notation throughout the paper. Unless specifed otherwise, we assume that $E$ is a Hausdorff sequentially complete locally convex space over the field of complex numbers, SCLCS for short. By $L(E)$ and $\circledast$ we denote the space consisting of all continuous linear mappings from $E$ into $E$ and the fundamental system of seminorms which defines the topology of $E$, respectively. Let $\mathscr{B}$ be the family consisting of all bounded subsets of $E$, and let $p_{\mathbf{B}}(T):=$ $\sup _{x \in \mathbf{B}} p(T x), p \in \circledast, \mathbf{B} \in \mathscr{B}, T \in L(E)$. Then $p_{\mathbf{B}}(\cdot)$ is a seminorm on $L(E)$ and the system $\left(p_{\mathbf{B}}\right)_{(p, \mathbf{B}) \in \circledast \times \mathscr{B}}$ induces the Hausdorff locally convex topology on $L(E)$. If $E$ is a Banach space, then we denote by $\|x\|$ the norm of an element $x \in E$. If $A$ is a closed linear operator acting on $E$, then the domain and range of $A$ will be denoted by $D(A)$ and $R(A)$, respectively. Since no confusion seems likely, we will identify $A$ with its graph. If $C \in L(E)$ is injective, then we define the $C$-resolvent set of $A, \rho_{C}(A)$ for short, by $\rho_{C}(A):=\{\lambda \in \mathbf{C} ; \lambda-A$ is injective and $\left.(\lambda-A)^{-1} C \in L(E)\right\}$. If $B$ is a closed linear operator with domain and range contained in $E$, then we define the set $\rho_{C}(B, A)$ by $\rho_{C}(B, A):=$ $\left\{\lambda \in \mathbf{C} ; \lambda B-A: D(A) \cap D(B) \rightarrow E\right.$ is injective and $\left.(\lambda B-A)^{-1} C \in L(E)\right\}$. By $I$ we denote the identity operator on $E$. The fractional power $\left(A B^{-1}\right)_{1 / 2}$, appearing in the paragraph directly after the proof of Theorem 2.6, will be understood in the sense of [21, Definition 2.9.24]; for further information concerning fractional powers of almost $C$-sectorial operators, the reader may consult [21, Section 2.9].

If $V$ is a general topological vector space, then a function $f: \Omega \rightarrow V$, where $\Omega$ is an open subset of $\mathbf{C}$, is said to be analytic if it is locally expressible in a neighborhood of any point $z \in \Omega$ by a uniformly convergent power series with coefficients in $V$. We refer the reader to [21, Section 1.1] and references cited there for the basic information about vector-valued analytic functions. In our approach the space $E$ is sequentially complete, so that the analyticity of a mapping $f: \Omega \rightarrow E(\varnothing \neq \Omega \subseteq \mathbf{C})$ is equivalent with its weak analyticity.

In the sequel of paper, we assume that $A$ and $B$ are two closed linear operators acting on $E$, as well as that $C \in L(E)$ is an injective operator satisfying $C A \subseteq A C$ and $C B \subseteq B C$. Sometimes we use the following condition on a scalar-valed function $K(\cdot)$ : 
(P1) $K(\cdot)$ is Laplace transformable, i.e., it is locally integrable on $[0, \infty)$ and there exists $\beta \in \mathbf{R}$ so that

$$
\tilde{K}(\lambda):=\mathscr{L}(K)(\lambda):=\lim _{b \rightarrow \infty} \int_{0}^{b} e^{-\lambda t} K(t) d t:=\int_{0}^{\infty} e^{-\lambda t} K(t) d t
$$

exists for all $\lambda \in \mathbf{C}$ with $\operatorname{Re} \lambda>\beta$.

By $\mathscr{F}$ and $\mathscr{F}^{-1}$ we denote the Fourier transform on $\mathbf{R}^{n}$ and its inverse transform, respectively. Given $\theta \in(0, \pi]$ and $d \in(0,1]$, define $\Sigma_{\theta}:=\{\lambda \in \mathbf{C}$ : $\lambda \neq 0,|\arg (\lambda)|<\theta\}$. The set consisting of all analytic functions from $\Sigma_{\theta}$ into $E$ is denoted by $\mathscr{A}\left(\Sigma_{\theta}: E\right)$. Further on, $\lfloor\beta\rfloor:=\sup \{n \in \mathbf{Z}: n \leq \beta\}$ and $\lceil\beta\rceil:=$ $\inf \{n \in \mathbf{Z}: \beta \leq n\}$. The Gamma function is denoted by $\Gamma(\cdot)$ and the principal branch is always used to take the powers; the convolution like mapping * is given by $f * g(t):=\int_{0}^{t} f(t-s) g(s) d s$. Set $g_{\zeta}(t):=t^{\zeta-1} / \Gamma(\zeta), 0^{\zeta}:=0 \quad(\zeta>0$, $t>0), g_{0}(t):=$ the Dirac $\delta$-distribution and $\mathbf{C}_{+}:=\{z \in \mathbf{C}: \Re z>0\}$.

Suppose $\beta>0$ and $\beta \notin \mathbf{N}$. Then the Liouville right-sided fractional derivative of order $\beta$ (see $[19,(2.3 .4)]$ for the scalar-valued case) is defined for those continuous functions $u:(0, \infty) \rightarrow E$ for which $\lim _{T \rightarrow \infty} \int_{s}^{T} g_{\lceil\beta\rceil-\beta}(t-s) u(t) d t=$ $\int_{s}^{\infty} g_{\lceil\beta\rceil-\beta}(t-s) u(t) d t$ exists and defines a $\lceil\beta\rceil$-times continuously differentiable function on $(0, \infty)$, by

$$
\mathbf{D}_{-}^{\beta} u(s):=(-1)^{\lceil\beta\rceil} \frac{d^{\lceil\beta\rceil}}{d s^{\lceil\beta\rceil}} \int_{s}^{\infty} g_{\lceil\beta\rceil-\beta}(t-s) u(t) d t, \quad s>0 .
$$

We define the modified Liouville right-sided fractional derivative of order $\beta$, $D_{-}^{\beta} u(s)$ shortly, for those continuously differentiable functions $u:(0, \infty) \rightarrow E$ for which $\lim _{T \rightarrow \infty} \int_{s}^{T} g_{\lceil\beta\rceil-\beta}(t-s) u^{\prime}(t) d t=\int_{s}^{\infty} g_{\lceil\beta\rceil-\beta}(t-s) u^{\prime}(t) d t$ exists and defines a $\lceil\beta-1\rceil$-times continuously differentiable function on $(0, \infty)$, by

$$
D_{-}^{\beta} u(s):=(-1)^{\lceil\beta\rceil} \frac{d^{\lceil\beta-1\rceil}}{d s^{\lceil\beta-1\rceil}} \int_{s}^{\infty} g_{\lceil\beta\rceil-\beta}(t-s) u^{\prime}(t) d t, \quad s>0
$$

if $\beta=n \in \mathbf{N}$, then $\mathbf{D}_{-}^{n} u$ and $D_{-}^{n} u$ are defined for all $n$-times continuously differentiable functions $u(\cdot)$ on $(0, \infty)$, by $\mathbf{D}_{-}^{n} u:=D_{-}^{n} u:=(-1)^{n} d / d^{n}$, where $d / d^{n}$ denotes the usual derivative operator of order $n$ (cf. also [19, (2.3.5)]).

The Mittag-Leffler function $E_{\beta, \gamma}(z)(\beta>0, \gamma \in \mathbf{R})$ is defined by

$$
E_{\beta, \gamma}(z):=\sum_{k=0}^{\infty} \frac{z^{k}}{\Gamma(\beta k+\gamma)}, \quad z \in \mathbf{C} .
$$


In this place, we assume that $1 / \Gamma(\beta k+\gamma)=0$ if $\beta k+\gamma \in-\mathbf{N}_{0}$. Set $E_{\beta}(z):=$ $E_{\beta, 1}(z), z \in \mathbf{C}$. For further information about the Mittag-Leffler functions, fractional calculus and non-degenerate fractional differential equations, see [4], [11], [19], [21], [36], [38] and references cited there. The basic information on abstract Volterra integro-differential equations in Banach and locally convex spaces can be obtained by consulting [37] and [21].

Assume that $n \in \mathbf{N}$ and $i A_{j}, 1 \leq j \leq n$ are commuting generators of bounded $C_{0}$-groups on a Banach space $E$. Set $\mathbf{A}:=\left(A_{1}, \ldots, A_{n}\right), \mathbf{A}^{\eta}:=A_{1}^{\eta_{1}} \cdots A_{n}^{\eta_{n}}$ for any $\eta=\left(\eta_{1}, \ldots, \eta_{n}\right) \in \mathbf{N}_{0}^{n}$, and denote by $\mathscr{S}\left(\mathbf{R}^{n}\right)$ the Schwartz space of rapidly decreasing functions on $\mathbf{R}^{n}$. Let $k=1+\lfloor n / 2\rfloor$. For every $\xi=\left(\xi_{1}, \ldots, \xi_{n}\right) \in \mathbf{R}^{n}$ and $u \in \mathscr{F} L^{1}\left(\mathbf{R}^{n}\right)=\left\{\mathscr{F} f: f \in L^{1}\left(\mathbf{R}^{n}\right)\right\}$, we set $|\xi|:=\left(\sum_{j=1}^{n} \xi_{j}^{2}\right)^{1 / 2}, \quad(\xi, A):=$ $\sum_{j=1}^{n} \xi_{j} A_{j}$ and

$$
u(A) x:=\int_{\mathbf{R}^{n}} \mathscr{F}^{-1} u(\xi) e^{-i(\xi, A)} x d \xi, \quad x \in E
$$

Then $u(A) \in L(E), u \in \mathscr{F} L^{1}\left(\mathbf{R}^{n}\right)$ and there exists a finite constant $M \geq 1$ such that

$$
\|u(A)\| \leq M\left\|\mathscr{F}^{-1} u\right\|_{L^{1}\left(\mathbf{R}^{n}\right)}, \quad u \in \mathscr{F} L^{1}\left(\mathbf{R}^{n}\right) .
$$

Let $N \in \mathbf{N}$, and let $P(x)=\sum_{|\eta| \leq N} a_{\eta} x^{\eta}, x \in \mathbf{R}^{n}$ be a complex polynomial. Then we define

$$
P(A):=\sum_{|\eta| \leq N} a_{\eta} A^{\eta} \quad \text { and } \quad E_{0}:=\left\{\phi(A) x: \phi \in \mathscr{S}\left(\mathbf{R}^{n}\right), x \in E\right\} .
$$

We know that the operator $P(A)$ is closable and that the following holds:

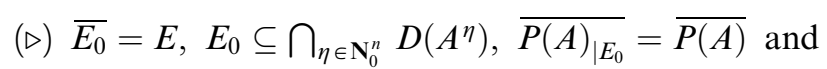
$\phi(A) P(A) \subseteq P(A) \phi(A)=(\phi P)(A), \phi \in \mathscr{S}\left(\mathbf{R}^{n}\right)$.

Assuming that $E$ is a function space on which translations are uniformly bounded and strongly continuous, the obvious choice for $A_{j}$ is $-i \partial / \partial x_{j}$ (notice also that $E$ can be consisted of functions defined on some bounded domain). If $P(x)=\sum_{|\eta| \leq N} a_{\eta} x^{\eta}, x \in \mathbf{R}^{n}$ and $E$ is such a space (for example, $L^{p}\left(\mathbf{R}^{n}\right.$ ) with $p \in[1, \infty), C_{0}\left(\mathbf{R}^{n}\right)$ or $\left.B U C\left(\mathbf{R}^{n}\right)\right)$, then it is not difficult to prove that $\overline{P(A)}$ is nothing else but the operator $\sum_{|\eta| \leq N} a_{\eta}(-i)^{|\eta|} \partial^{|\eta|} / \partial x_{1}^{\eta_{1}} \cdots \partial x_{n}^{\eta_{n}} \equiv \sum_{|\eta| \leq N} a_{\eta} D^{\eta}$, acting with its maximal distributional domain. For further information concerning the functional calculus for commuting generators of $C_{0}$-groups, see [9], [21] and [46]. 


\section{Degenerate $C$-Regularized Semigroups of Growth Order $r>0$ and Their Applications in the Study of Incomplete Degenerate Abstract Cauchy Problems}

In a joint paper with V. Fedorov [29], the author has recently analyzed a class of abstract degenerate muti-term fractional differential equations with Caputo derivatives. The integral computation from [29, Remark 2.2(vii)] has motivated us to introduce the following definition (cf. also the proof of [39, Lemma 1.4] and the equation (2.5) below).

Definition 2.1. Suppose that $B$ is a closed linear operator on $E$ and $C \in L(E)$ is an injective operator. An operator family $(T(t))_{t>0} \subseteq L(E)$ is said to be a pre- $(B, C)$-regularized semigroup of growth order $r>0$ iff $R(T(t)) \subseteq D(B)$, $t>0$ and the following holds:

(a) $T(t+s) C=T(t) B T(s)$ for all $t, s>0$,

(b) for every $x \in E$, the mapping $t \mapsto T(t) x, t>0$ is continuous, and

(c) the family $\left\{t^{r} T(t): t \in(0,1]\right\} \subseteq L(E)$ is equicontinuous.

$(T(t))_{t>0} \subseteq L(E)$ is said to be a $(B, C)$-regularized semigroup of growth order $r>0$ iff, in addition to (a)-(c), we have that:

(d) for every $x \in E$, the mapping $t \mapsto B T(t) x, t>0$ is continuous, and

(e) the family $\left\{t^{r} B T(t): t \in(0,1]\right\} \subseteq L(E)$ is equicontinuous.

The notion of an analytic (pre-) $(B, C)$-regularized semigroup of growth order $r>0$ is introduced in the following definition.

Definition 2.2. Suppose that $B$ is a closed linear operator on $E$ and $C \in L(E)$ is an injective operator.

(i) Suppose $0<\gamma \leq \pi / 2,(T(t))_{t>0}$ is a pre- $(B, C)$-regularized semigroup of growth order $r>0$, and the mapping $t \mapsto T(t) x, t>0$ has an analytic extension to the sector $\Sigma_{\gamma}$, denoted by the same symbol $(x \in E)$. If there exists $\omega \in \mathbf{R}(\omega=0)$ such that, for every $\delta \in(0, \gamma)$, the family $\left\{|z|^{r} e^{-\omega \Re z} T(z): z \in \Sigma_{\delta}\right\} \subseteq L(E)$ is equicontinuous, then $(T(z))_{z \in \Sigma_{y}} \subseteq L(E)$ is said to be an (equicontinuous) analytic pre- $(B, C)$-regularized semigroup of growth order $r$.

(ii) Suppose $0<\gamma \leq \pi / 2, \quad(T(t))_{t>0}$ is a $(B, C)$-regularized semigroup of growth order $r>0$, the mappings $t \mapsto T(t) x, t>0$ and $t \mapsto B T(t) x, t>0$ admit analytic extensions to the sector $\Sigma_{\gamma}$, denoted by the same symbols $(x \in E)$, and there exists $\omega \in \mathbf{R}(\omega=0)$ such that, for every $\delta \in(0, \gamma)$, the 
families $\left\{|z|^{r} e^{-\omega \Re z} T(z): z \in \Sigma_{\delta}\right\} \subseteq L(E)$ and $\left\{|z|^{r} e^{-\omega \Re z} B T(z): z \in \Sigma_{\delta}\right\} \subseteq$ $L(E)$ are equicontinuous. Then $(T(z))_{z \in \Sigma} \subseteq L(E)$ is said to be an (equicontinuous) analytic $(B, C)$-regularized semigroup of growth order $r$.

REMARK 2.3. (i) Our assumption $R(T(t)) \subseteq D(B), t>0$ immediately implies that $B T(t)$ is a closed linear operator for all $t>0$. In the case that $E$ is a webbed bornological space (this, in particular, holds if $E$ is a Fréchet space; cf. [32]), then the above implies by the closed graph theorem that $B T(t) \in L(E)$ for all $t>0$.

(ii) If $(T(t))_{t>0}$ is a $(B, C)$-regularized semigroup of growth order $r>0$ satisfying additionally that the preassumption $B T(t) x=0, t>0$ implies $x=0$, then $(B T(t))_{t>0} \subseteq L(E)$ is a $C$-regularized semigroup of growth order $r>0$ (in the sense of [6, Definition 3.4]). A similar statement can be formulated for the class of analytic $(B, C)$-regularized semigroups.

The notion of integral generator of $(T(t))_{t>0}$ and the notion of complete infinitesimal generator of $(T(t))_{t>0}$ can be very simply introduced provided that $B=I([6])$. Unfortunately, the situation is much more complicated in the case that $B \neq I$, where we probably should observe, before doing anything else, that $\mathscr{A}_{B}:=\left\{(x, y) \in E \times E: B T(t) x-B T(s) x=B \int_{s}^{t} T(r) y d r\right.$ for all $t, s>0$ with $t \geq s\}$ is a multivalued linear operator in the sense of [13, Definition, p. 21]; after that, we can try to define the integral generator of $(T(t))_{t>0}$ and the complete infinitesimal generator of $(T(t))_{t>0}$ as some appropriately chosen single-valued branches of $\mathscr{A}_{B}$ (cf. [13, p. 22, 1. 13-14] and [33, Proposition 1.6.4]). In our opinion, the ideal option to define generators of $(T(t))_{t>0}$ does not really exists in degenerate case and, because of that, we shall skip all related details concerning these questions for the sake of brevity and better exposition.

We need to introduce the following condition

$(\mathrm{H}): A$ and $B$ are closed linear operators on $E, C \in L(E)$ is injective, $0 \leq \omega<\pi, q \in \mathbf{R}, \mathbf{C} \backslash \overline{\Sigma_{\omega}} \subseteq \rho_{C}(B, A)$, the families

$$
\begin{array}{r}
\left\{\left(|\lambda|^{-1}+|\lambda|^{q}\right)^{-1}(\lambda B-A)^{-1} C: \lambda \notin \Sigma_{\omega^{\prime}}\right\} \subseteq L(E) \text { and } \\
\left\{\left(|\lambda|^{-1}+|\lambda|^{q}\right)^{-1} B(\lambda B-A)^{-1} C: \lambda \notin \Sigma_{\omega^{\prime}}\right\} \subseteq L(E)
\end{array}
$$

are equicontinuous for every $\omega<\omega^{\prime}<\pi$, as well as the mappings $\lambda \mapsto(\lambda B-A)^{-1} C x, \quad \lambda \in \mathbf{C} \backslash \overline{\Sigma_{\omega}}$ and $\lambda \mapsto B(\lambda B-A)^{-1} C x, \quad \lambda \in \mathbf{C} \backslash \overline{\Sigma_{\omega}}$ are continuous for every fixed element $x \in E$. 
Since we have assumed that $C A \subseteq A C$ and $C B \subseteq B C$, the analysis contained in [29, Remark 2.2(vii)] shows that the validity of condition $(\mathrm{H})$ implies that the mappings $\lambda \mapsto(\lambda B-A)^{-1} C x, \lambda \in \mathbf{C} \backslash \overline{\Sigma_{\omega}}$ and $\lambda \mapsto B(\lambda B-A)^{-1} C x$, $\lambda \in \mathbf{C} \backslash \overline{\Sigma_{\omega}}$ are analytic, indeed, for every fixed element $x \in E$. By $\mathscr{M}_{B, C, q}$ we denote the class consisting of all closed linear operators $A^{\prime}$ on $E$, satisfying that the families $\left\{\left(|\lambda|^{-1}+|\lambda|^{q}\right)^{-1}\left(\lambda B-A^{\prime}\right)^{-1} C: \lambda \in(-\infty, 0)\right\} \subseteq L(E)$ and $\left\{\left(|\lambda|^{-1}+|\lambda|^{q}\right)^{-1} B\left(\lambda B-A^{\prime}\right)^{-1} C: \lambda \in(-\infty, 0)\right\} \subseteq L(E)$ are equicontinuous, as well as that the mappings $\lambda \mapsto\left(\lambda B-A^{\prime}\right)^{-1} C x, \lambda \in(-\infty, 0)$ and $\lambda \mapsto B\left(\lambda B-A^{\prime}\right)^{-1} C x$, $\lambda \in(-\infty, 0)$ are continuous for every fixed element $x \in E$.

Following A. V. Balakrishnan [3], we introduce the function $f_{t, \gamma}(\lambda)$ by

$$
\begin{aligned}
f_{t, \gamma}(\lambda) & :=\frac{1}{\pi} e^{-t \lambda^{\gamma} \cos \pi \gamma} \sin \left(t \lambda^{\gamma} \sin \pi \gamma\right) \\
& =\frac{1}{2 \pi i}\left(e^{-t \lambda^{\gamma} e^{-i \pi \gamma}}-e^{-t \lambda^{\gamma} e^{i \pi \gamma}}\right), \quad \lambda>0(t>0, \gamma \in(0,1 / 2)) .
\end{aligned}
$$

This function enjoys the following properties ([31]):

1. $\left|f_{t, \gamma}(\lambda)\right| \leq \pi^{-1} e^{-\lambda^{\gamma} \epsilon_{t, \gamma}}, \lambda>0$, where $\epsilon_{t, \gamma}:=t \cos \pi \gamma>0$.

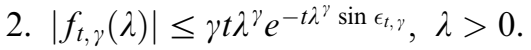

3. $\int_{0}^{\infty} \lambda^{n} f_{t, \gamma}(\lambda) d \lambda=0, n \in \mathbf{N}_{0}$.

Set

$$
H_{n}(\omega, z):=\frac{d^{n}}{d z^{n}} \exp \left(-\omega z^{\gamma}\right), \quad \omega \in \mathbf{C}, z \in \mathbf{C} \backslash(-\infty, 0],
$$

and, if that makes any sense,

$$
W_{\gamma}(t) x:=\int_{0}^{\infty} f_{t, \gamma}(\lambda)(\lambda B+A)^{-1} C x d \lambda, \quad t>0, x \in E(\gamma \in(0,1 / 2)) .
$$

Then the function $H_{n}(\omega, z)$ is analytic in $\mathbf{C} \backslash(-\infty, 0]$ for every fixed number $\omega$, and entire in $\mathbf{C}$ for every fixed number $z$ (cf. also the proof of [31, Proposition 3.5]).

Our next task will be to enquire into the possibility to transmit the assertion of [21, Theorem 2.9.48] to abstract degenerate differential equations (it is clear that some parts of this theorem cannot be so simply formulated for degenerate differential equations because we do not know what would be the fractional power $A_{z}\left(z \in \mathbf{C}_{+}\right)$in the newly arisen situation). Concerning this question, we will state and prove two theorems, Theorem 2.4 and Theorem 2.5. In Theorem 
2.4, we will investigate the properties of operator family $\left(W_{\gamma}(t)\right)_{t>0}$ in more detail.

Theorem 2.4. Let $0<\gamma<1 / 2$, and let $A \in \mathscr{M}_{B, C, q}$ where $q+\gamma>-1$. Suppose that $C A \subseteq A C$ and $C B \subseteq B C$. Then the families $\left\{\left(1+t^{-(q+1) / \gamma}\right)^{-1} W_{\gamma}(t)\right.$ : $t>0\} \subseteq L(E)$ and $\left\{\left(1+t^{-(q+1) / \gamma}\right)^{-1} B W_{\gamma}(t): t>0\right\} \subseteq L(E)$ are equicontinuous, and there exists an operator family $\left(\mathbf{W}_{\gamma}(z)\right)_{z \in \Sigma_{(\pi / 2)-\gamma \pi}} \subseteq L(E)$, resp., $\left(\mathbf{W}_{\gamma, B}(z)\right)_{z \in \Sigma_{(\pi / 2)-\gamma \pi}} \subseteq L(E)$, such that, for every $x \in E$, the mapping $z \mapsto \mathbf{W}_{\gamma}(z) x$, $z \in \Sigma_{(\pi / 2)-\gamma \pi}, \quad$ resp., $\quad z \mapsto \mathbf{W}_{\gamma, B}(z) x, \quad z \in \Sigma_{(\pi / 2)-\gamma \pi}$, is analytic as well as that $\mathbf{W}_{\gamma}(t)=W_{\gamma}(t), t>0$, resp., $\mathbf{W}_{\gamma, B}(t)=B W_{\gamma}(t), t>0$ (in the sequel, we will not make any difference between $\mathbf{W}_{\gamma}(\cdot)$ and $W_{\gamma}(\cdot), \mathbf{W}_{\gamma, B}(\cdot)$ and $\left.W_{\gamma, B}(\cdot)\right)$. Furthermore, the following holds:

(i) $W_{\gamma}\left(z_{1}\right) B W_{\gamma}\left(z_{2}\right)=W_{\gamma}\left(z_{1}+z_{2}\right) C$ for all $z_{1}, z_{2} \in \Sigma_{(\pi / 2)-\gamma \pi}$.

(ii) Let $-1-\gamma<q \leq-1$. If $(\lambda B-A)^{-1} C A \subseteq A(\lambda B-A)^{-1} C, \lambda \in \rho_{C}(A, B)$ and $x \in \overline{D(A)}$, then

$$
\lim _{z \rightarrow 0, z \in \Sigma_{(\pi / 2)-\gamma \pi-\epsilon}} B W_{\gamma}(z) x=C x, \quad \epsilon \in(0,(\pi / 2)-\gamma \pi)
$$

if, in addition to this, the condition $(\mathrm{H})$ holds, then we can extend the operator family $W_{\gamma}(\cdot)$ to the sector $\Sigma_{\pi / 2-\omega \gamma}$ and the limit equality (2.1) remains true for each $x \in \overline{D(A)}$, with the number $(\pi / 2)-\gamma \pi$ replaced by $(\pi / 2)-\omega \gamma$.

(iii) Let $q>-1$. Then $\left(W_{\gamma}(z)\right)_{z \in \Sigma_{(\pi / 2)-\gamma \pi}}$ is an equicontinuous analytic $(B, C)$ regularized semigroup of growth order $(q+1) / \gamma$. If, additionally, the condition $(\mathrm{H})$ holds, then $\left(W_{\gamma}(t)\right)_{t>0}$ can be extended to an equicontinuous analytic $(B, C)$-regularized semigroup $\left(\mathbf{W}_{\gamma}(z)\right)_{z \in \Sigma_{(\pi / 2)-\gamma \omega}}$ of growth order $(q+1) / \gamma$. Suppose that the operator $B$ is injective, $x \in D\left(\left(B^{-1} A\right)^{\lfloor q+2\rfloor}\right)$ $\cap D(B) \quad$ and $\quad(\lambda B-A)^{-1} C B \subseteq B(\lambda B-A)^{-1} C \quad\left(\lambda \in \rho_{C}(A, B)\right)$. Then $\lim _{z \rightarrow 0, z \in \Sigma_{(\pi / 2)-\gamma \pi-\epsilon}} B W_{\gamma}(z) x=C x, \quad \epsilon \in(0,(\pi / 2)-\gamma \pi)$; if, in addition to this, the condition $(\mathrm{H})$ holds, then the above limit equality remains true with the number $(\pi / 2)-\gamma \pi$ replaced by $(\pi / 2)-\omega \gamma$.

(iv) Suppose that $q<0$ and $x \in D(A) \cap D(B)$. Then $\lim _{z \rightarrow 0, z \in \Sigma_{(\pi / 2)-\gamma \pi-\epsilon}} W_{\gamma}(z) B x$ $=C x, \epsilon \in(0,(\pi / 2)-\gamma \pi)$; if, in addition to this, the condition $(\mathrm{H})$ holds, then the above limit equality remains true with the number $(\pi / 2)-\gamma \pi$ replaced by $(\pi / 2)-\omega \gamma$.

(v)(v.1) Let $q+\gamma \geq 0, \quad z_{0} \in \mathbf{C}_{+}$, let $B$ be injective, $x \in D\left(\left(B^{-1} A\right)^{\lfloor q+\gamma\rfloor+2}\right) \cap$ $D(B)$, and let $(\lambda B-A)^{-1} C B \subseteq B(\lambda B-A)^{-1} C\left(\lambda \in \rho_{C}(A, B)\right)$. Then 


$$
\begin{aligned}
\lim _{z \rightarrow 0, z \in \Sigma_{(\pi / 2)-\gamma \pi-\epsilon}} \frac{B W_{\gamma}(z) x-C x}{z} & =-z_{0}^{\gamma} C x+\sum_{k=2}^{\lfloor q+\gamma\rfloor+2} \frac{(-1)^{k-1}}{(k-1) !} H_{k-1}\left(0, z_{0}\right)\left(z_{0}-B^{-1} A\right)^{k-1} C x \\
& -\sin (\pi \gamma) \int_{0}^{\infty} \lambda^{\gamma} \frac{B(\lambda+A)^{-1} C\left(z_{0}-B^{-1} A\right)^{\lfloor q+\gamma\rfloor+2} x}{\left(\lambda+z_{0}\right)^{\lfloor q+\gamma\rfloor+2}} d \lambda \\
& \epsilon \in(0,(\pi / 2)-\gamma \pi)
\end{aligned}
$$

if the condition $(\mathrm{H})$ holds, then the formula (2.2) remains true with the number $(\pi / 2)-\gamma \pi$ replaced by $(\pi / 2)-\omega \gamma$.

(v.2) Let $q+\gamma<0, z_{0} \in \mathbf{C}_{+}$and $x \in D(A) \cap D(B)$. Then

$$
\begin{aligned}
& \lim _{z \rightarrow 0, z \in \Sigma_{(\pi / 2)-\gamma \pi-\epsilon}} \frac{W_{\gamma}(z) B x-C x}{z} \\
& =-z_{0}^{\gamma} C x-\sin (\pi \gamma) \int_{0}^{\infty} \lambda^{\gamma} \frac{(\lambda+A)^{-1} C\left(z_{0} B-A\right) x}{\left(\lambda+z_{0}\right)} d \lambda, \\
& \quad \epsilon \in(0,(\pi / 2)-\gamma \pi) ;
\end{aligned}
$$

if the condition $(\mathrm{H})$ holds, then the formula (2.3) remains true with the number $(\pi / 2)-\gamma \pi$ replaced by $(\pi / 2)-\omega \gamma$.

ProOF. The existence of operator families $\left(\mathbf{W}_{\gamma}(z)\right)_{z \in \Sigma_{(\pi / 2)-\gamma \pi}}$ and $\left(\mathbf{W}_{\gamma, B}(z)\right)_{z \in \Sigma_{(\pi / 2)-\gamma \pi}}$, satisfying the properties stated in the first part of formulation of theorem, before the assertion (i), follows similarly as in the case that $B=I$. Suppose $\delta \in(0,(\pi / 2)-\pi \gamma)$ and $r \in \circledast$. Arguing as in the proof of [21, Theorem 2.9.48], we obtain that there exist $r^{\prime} \in \circledast$ and $m>0$ such that, for every $x \in E$ and $z \in \Sigma_{\delta}$, we have

$$
\begin{aligned}
& r\left(W_{\gamma}(z) x\right) \leq m\left(1+|z|^{-(q+1) / \gamma}\right) r^{\prime}(x) \quad \text { and } \\
& r\left(B W_{\gamma}(z) x\right) \leq m\left(1+|z|^{-(q+1) / \gamma}\right) r^{\prime}(x) .
\end{aligned}
$$

The semigroup property stated in (i) can be proved by using the resolvent equation

$$
\begin{aligned}
(z B & +A)^{-1} C^{2} x-\left(z^{\prime} B+A\right)^{-1} C^{2} x \\
& =\left(z^{\prime}-z\right)(z B+A)^{-1} C B\left(z^{\prime} B+A\right)^{-1} C x, \quad z, z^{\prime} \in \rho_{C}(A, B), x \in E,
\end{aligned}
$$

and direct computations, similar to those appearing in [3, Section 3]. Therefore, $\left(W_{\gamma}(z)\right)_{z \in \Sigma_{(\pi / 2)-\gamma \pi}}$ is an equicontinuous analytic $(B, C)$-regularized semigroup of 
growth order $(q+1) / \gamma$, provided that $q>-1$. Suppose temporarily that the operator $B$ is injective and $B(\lambda B-A)^{-1} C \subseteq(\lambda B-A)^{-1} C B\left(\lambda \in \rho_{C}(A, B)\right)$. Then the following equality holds

$$
\left(\lambda+B^{-1} A\right)^{-1} C x=B(\lambda B+A)^{-1} C x, \quad \lambda>0, x \in D(B) .
$$

Combined with the identity $[31$, p. $212,1.8],(2.6)$ implies that, for every $\lambda>0$ and $z_{0} \in \mathbf{C}_{+}$, we have

$$
\begin{aligned}
B(\lambda B & +A)^{-1} C x \\
& =\sum_{j=1}^{\lfloor q+2\rfloor} \frac{\left(z_{0}-B^{-1} A\right)^{j-1} C x}{\left(\lambda+z_{0}\right)^{j}}+\frac{B(\lambda B+A)^{-1} C\left(z_{0}-B^{-1} A\right)^{\lfloor q+2\rfloor} C x}{\left(\lambda+z_{0}\right)^{\lfloor q+2\rfloor}},
\end{aligned}
$$

so that the proof of limit equality in (iii), on proper subsectors of $\Sigma_{(\pi / 2)-\gamma \pi}$, follows in almost the same way as in the proof of [31, Proposition 3.5]. Using the proof of [21, Theorem 2.9.48(v)], we obtain similarly that the limit equality in (v.1) holds on proper subsectors of $\Sigma_{(\pi / 2)-\gamma \pi}$. We continue the proof of (iii). Suppose that $\epsilon>0$ is sufficiently small. Let $q>-1$, and let the condition (H) hold. Then one can take numbers $\theta_{1} \in(0, \pi-\omega)$ and $\theta_{2} \in(\omega-\pi, 0)$ such that $(\pi / 2)-\gamma \omega>(\pi / 2)-\gamma \pi+\gamma \theta_{1}>(\pi / 2)-\gamma \omega-\epsilon$ and $\omega \gamma-(\pi / 2)+\epsilon>\gamma \pi-$ $(\pi / 2)+\gamma \theta_{2}>\omega \gamma-(\pi / 2)$. Set, for every $\theta \in(\omega-\pi, \pi-\omega)$,

$$
W_{\theta, \gamma}(z) x:=\int_{0}^{\infty} f_{z, \gamma}(\lambda)\left(\lambda B+e^{i \theta} A\right)^{-1} C x d \lambda, \quad x \in E, z \in \Sigma_{(\pi / 2)-\gamma \pi} .
$$

Then it can be simply verified that $\left(W_{\theta, \gamma}(z)\right)_{z \in \Sigma_{(\pi / 2)-\gamma \pi}}$ is an equicontinuous analytic $(B, C)$-regularized semigroup of growth order $(q+1) / \gamma$. Define, for every $x \in E$,

$$
W_{\gamma}(z) x:=\left\{\begin{array}{l}
W_{\gamma}(z) x, \quad \text { if } z \in \Sigma_{(\pi / 2)-\gamma \pi} \\
W_{\theta_{1}, \gamma}\left(z e^{-i \theta_{1} \gamma}\right) x, \quad \text { if } z \in e^{i \theta_{1} \gamma \Sigma_{(\pi / 2)-\gamma \pi}} \\
W_{\theta_{2}, \gamma}\left(z e^{-i \theta_{2} \gamma}\right) x, \quad \text { if } z \in e^{i \theta_{2} \gamma \Sigma_{(\pi / 2)-\gamma \pi}}
\end{array}\right.
$$

An elementary application of Cauchy's formula shows that $W_{\gamma}(z)=W_{\theta_{1}, \gamma}\left(z e^{-i \theta_{1} \gamma}\right)$, if $z \in \Sigma_{(\pi / 2)-\gamma \pi} \cap e^{i \theta_{1} \gamma} \Sigma_{(\pi / 2)-\gamma \pi}$, and $W_{\gamma}(z)=W_{\theta_{2}, \gamma}\left(z e^{-i \theta_{2} \gamma}\right)$, if $z \in \Sigma_{(\pi / 2)-\gamma \pi} \cap$ $e^{i \theta_{2} \gamma} \Sigma_{(\pi / 2)-\gamma \pi}$, whence we may conclude that the operator family $\left(W_{\gamma}(z)\right)_{z \in \Sigma_{\pi / 2-\omega \gamma}}$ $\subseteq L(E)$ is well defined. We define the operator family $\left(B W_{\gamma}(z)\right)_{z \in \Sigma_{\pi / 2-\omega \gamma}} \subseteq L(E)$ similarly. Then it is checked at once that $\left(W_{\gamma}(z)\right)_{z \in \Sigma_{\pi / 2-\omega \gamma}}$ is an equicontinuous analytic $(B, C)$-regularized semigroup of growth order $(q+1) / \gamma$. The way of 
construction of $\left(W_{\gamma}(z)\right)_{z \in \Sigma_{\pi / 2-\omega \gamma}} \subseteq L(E)$ shows that the limit equality stated in (iii) continues to hold for each $x \in D\left(\left(B^{-1} A\right)^{\lfloor q+2\rfloor}\right) \cap D(B)$, with the number $(\pi / 2)-\gamma \pi$ replaced by $(\pi / 2)-\gamma \omega$ (if $q \leq-1$, then we define $\left(W_{\gamma}(z)\right)_{z \in \Sigma_{\pi / 2-\omega \gamma}} \subseteq$ $L(E)$ and $\left(B W_{\gamma}(z)\right)_{z \in \Sigma_{\pi / 2-\omega \gamma}} \subseteq L(E)$ in the same way as above, showing also that any considered limit equality from (ii)-(v) continues to hold with the number $(\pi / 2)-\gamma \pi$ replaced by $(\pi / 2)-\gamma \omega)$. The proof of (iii) is therefore completed. Suppose now that $-1-\gamma<q \leq-1$. Then an insignificant modification of the proof of [30, Theorem 5.5.1(iv)] shows that $\lim _{t \rightarrow 0+} B W_{\gamma}(t) x=$ $C x$, provided that $x \in E$ satisfies $\lim _{\lambda \rightarrow+\infty} \lambda B(\lambda B+A)^{-1} C x=C x$. Since we have assumed that $A$ commutes with $(\lambda B+A)^{-1} C\left(\lambda \in \rho_{C}(A, B)\right)$, and that $\lambda B(\lambda B+A)^{-1} C x-C x=A(\lambda B+A)^{-1} C x=(\lambda B+A)^{-1} C A x, \quad x \in D(A), \quad \lambda>0$, one has $\lim _{t \rightarrow 0+} B W_{\gamma}(t) x=C x, x \in D(A)$. Using (2.4), it readily follows that $\lim _{t \rightarrow 0+} B W_{\gamma}(t) x=C x, x \in \overline{D(A)}$, so that the limit equality (2.1) follows from an application of [22, Theorem 3.4(ii)]. The limit equalities stated in (iv) and (v.2), on proper subsectors of $\Sigma_{(\pi / 2)-\gamma \pi}$, can be proved by using the equality

$$
(\lambda B+A)^{-1} C B x=\frac{C x}{\lambda+z_{0}}+\frac{(\lambda B+A)^{-1} C}{\lambda+z_{0}}\left(z_{0} B x-A x\right),
$$

which holds for $z_{0} \in \mathbf{C}_{+}, \lambda>0, x \in D(A) \cap D(B)$, and a slight modification of the proofs of [31, Lemma 3.4, Proposition 3.5]. The proof of the theorem is thereby complete.

In Theorem 2.5, we use the same terminology as in the formulation and proof of Theorem 2.4. We continue our previous analysis by investigating the existence of solutions of abstract degenerate Cauchy problems $\left(F P_{\alpha_{1}, \beta_{1}, \theta}\right)$ and $\left(F P_{\alpha_{1}, \beta_{1}, \theta}\right)^{\prime}$.

THEOREM 2.5. Let $0<\gamma<1 / 2$, and let $A \in \mathscr{M}_{B, C, q}$ where $q+\gamma>-1$. Suppose that $C A \subseteq A C, \quad C B \subseteq B C, \quad \alpha_{1} \geq 0, \quad \beta_{1} \geq 0, \quad \alpha_{1}+\beta_{1}=1 / \gamma$ and $\theta \in$ $(\gamma \pi-(\pi / 2),(\pi / 2)-\gamma \pi)$. Then the following holds:

(i) Let $q>-1$. Denote by $\Omega_{\theta, \gamma}$, resp. $\Psi_{\gamma}$, the set consisting of those elements $x \in E$ for which $\lim _{s \rightarrow 0+} B W_{\gamma}\left(s e^{i \theta}\right) x=C x$, resp., $\lim _{z \rightarrow 0, z \in \Sigma_{(\pi / 2)-\gamma \pi-\epsilon}} B W_{\gamma}(z) x$ $=C x$ for all $\epsilon \in(0,(\pi / 2)-\gamma \pi)$. Then, for every $x \in \Omega_{\theta, \gamma}$, the incomplete abstract degenerate Cauchy problem $\left(F P_{\alpha_{1}, \beta_{1}, \theta}\right)$ has a solution $u(s)=$ $W_{\gamma}\left(s^{i \theta}\right) x, \quad s>0$, which can be analytically extended to the sector $\Sigma_{(\pi / 2)-\gamma \pi-|\theta|}$. If, additionally, $x \in \Psi_{\gamma}$, then for every $\delta \in(0,(\pi / 2)-\gamma \pi-|\theta|)$ and $j \in \mathbf{N}_{0}$, we have that the set $\left\{z^{j} B u^{(j)}(z): z \in \Sigma_{\delta}\right\}$ is bounded in $E$. 
Assume that the condition $(\mathrm{H})$ holds. Then the solution $s \mapsto u(s), s>0$ can be analytically continued to the sector $\Sigma_{(\pi / 2)-\gamma \omega}$; if, in addition to this, $\lim _{z \rightarrow 0, z \in \Sigma_{(\pi / 2)-\gamma(\omega-\epsilon}} B W_{\gamma}(z) x=C x$ for all $\epsilon \in(0,(\pi / 2)-\gamma \omega)$, then for every $\delta \in(0,(\pi / 2)-\gamma \omega-|\theta|)$ and $j \in \mathbf{N}_{0}$, we have that the set $\left\{z^{j} B u^{(j)}(z): z \in \Sigma_{\delta}\right\}$ is bounded in $E$.

(ii) Let $-1-\gamma<q \leq-1, \quad(\lambda B-A)^{-1} C A \subseteq A(\lambda B-A)^{-1} C, \quad \lambda \in \rho_{C}(A, B)$, and let $x \in \overline{D(A)}$. Then the incomplete abstract degenerate Cauchy problem $\left(F P_{\alpha_{1}, \beta_{1}, \theta}\right)^{\prime}$ has a solution $u(s)=W_{\gamma}\left(s e^{i \theta}\right) x, s>0$, which can be analytically extended to the sector $\Sigma_{(\pi / 2)-\gamma \pi-|\theta|}$. Moreover, for every $\delta \in(0,(\pi / 2)-\gamma \pi-|\theta|)$ and $j \in \mathbf{N}_{0}$, the sets $\left\{|z|^{j}\left(1+|z|^{-(q+1) / \gamma}\right)^{-1} u^{(j)}(z)\right.$ : $\left.z \in \Sigma_{\delta}\right\}$ and $\left\{|z|^{j}\left(1+|z|^{-(q+1) / \gamma}\right)^{-1} B u^{(j)}(z): z \in \Sigma_{\delta}\right\}$ are bounded in E. If, additionally, the condition $(\mathrm{H})$ holds, then the above statements remain true with the number $(\pi / 2)-\gamma \pi$ replaced by $(\pi / 2)-\omega \gamma$.

Proof. The proof of theorem almost completely follows from the arguments used in those of [21, Theorem 2.9.48] and [6, Theorem 3.5(i)/ $\left.\mathrm{b}^{\prime}\right]$, and the only non-trivial thing that should be explained here is the way how we can prove that, for every $\delta \in(0,(\pi / 2)-\gamma \pi-|\theta|)$ and $j \in \mathbf{N}_{0}$, the solution $s \mapsto u(s)$, $s>0$ has the property that the sets $\left\{|z|^{j}\left(1+|z|^{-(q+1) / \gamma}\right)^{-1} u^{(j)}(z): z \in \Sigma_{\delta}\right\}$ and $\left\{|z|^{j}\left(1+|z|^{-(q+1) / \gamma}\right)^{-1} B u^{(j)}(z): z \in \Sigma_{\delta}\right\}$ are bounded in $E$ (cf. (ii)). In order to show this, observe first that for each $x \in E, z \in \Sigma_{\pi / 2-\gamma \pi}$ and $j \in \mathbf{N}$ we have

$$
\begin{aligned}
\frac{d^{j}}{d z^{j}} W_{\gamma}(z) x= & \frac{1}{2 \pi i} \int_{0}^{\infty}\left(-\lambda^{\gamma} e^{-i \pi \gamma}\right)^{j} e^{-z \lambda^{\gamma} e^{-i \pi \gamma}}(\lambda B+A)^{-1} C x d \lambda \\
& -\frac{1}{2 \pi i} \int_{0}^{\infty}\left(-\lambda^{\gamma} e^{i \pi \gamma}\right)^{j} e^{-z \lambda^{\gamma} e^{i \pi \gamma}}(\lambda B+A)^{-1} C x d \lambda .
\end{aligned}
$$

Hence, $\quad B \frac{d^{j}}{d z^{j}} W_{\gamma}(z) x=\frac{d^{j}}{d z^{j}} B W_{\gamma}(z) x \quad\left(x \in E, \quad z \in \Sigma_{\pi / 2-\gamma \pi}, \quad j \in \mathbf{N}\right)$, which implies that $B u^{(j)}(z)=(B u)^{(j)}(z) \quad\left(z \in \Sigma_{\pi / 2-\gamma \pi}, j \in \mathbf{N}\right)$. Having in mind this equality, the boundedness of sets $\left\{|z|^{j}\left(1+|z|^{-(q+1) / \gamma}\right)^{-1} u^{(j)}(z): z \in \Sigma_{\delta}\right\}$ and $\left\{|z|^{j}(1+\right.$ $\left.\left.|z|^{-(q+1) / \gamma}\right)^{-1} B u^{(j)}(z): z \in \Sigma_{\delta}\right\}$ can be proved by using (2.7) and a direct computation involving the estimates used in the proof of [20, Theorem 1.4.15].

The interested reader may try to reconsider the assertions of [21, Theorem 2.9.39, Theorem 2.9.40, Theorem 2.9.58, Theorem 2.9.60] for degenerate differential equations. Now we will focus our attention on the assertion of [21, Theorem 2.9.51(iii)]. 
THEOREM 2.6. Suppose that the condition $(\mathrm{H})$ holds, as well as that $C A \subseteq A C$ and $C B \subseteq B C$.

(i) Let $-1<q<(-1) / 2$, and let $(\lambda B-A)^{-1} C A \subseteq A(\lambda B-A)^{-1} C \quad(\lambda \in$ $\left.\rho_{C}(A, B)\right)$. Then, for every $x \in D(A)$, the incomplete abstract degenerate Cauchy problem $\left(P_{2, q, B}\right)$ has a solution $u(z)\left(z \in \Sigma_{(\pi / 2)-(\omega / 2)}\right)$. Moreover, for every $\delta \in(0,(\pi / 2)-(\omega / 2))$ and $j \in \mathbf{N}_{0}$, we have that the sets $\left\{|z|^{j}\left(1+|z|^{-(2 q+2)}\right)^{-1} u^{(j)}(z): z \in \Sigma_{\delta}\right\}$ and $\left\{|z|^{j}\left(1+|z|^{-(2 q+2)}\right)^{-1}(B u)^{(j)}(z)\right.$ : $\left.z \in \Sigma_{\delta}\right\}$ are bounded in $E$.

(ii) Let $-1 \geq q>(-3) / 2$, and let $(\lambda B-A)^{-1} C A \subseteq A(\lambda B-A)^{-1} C \quad(\lambda \in$ $\left.\rho_{C}(A, B)\right)$. Then, for every $x \in \overline{D(A)}$, the problem $\left(P_{2, q, B}\right)$ has a solution $u(z) \quad\left(z \in \Sigma_{(\pi / 2)-(\omega / 2)}\right)$. Moreover, for every $\delta \in(0,(\pi / 2)-(\omega / 2))$ and $j \in \mathbf{N}_{0}$, we have that the sets $\left\{|z|^{j}\left(1+|z|^{-(2 q+2)}\right)^{-1} u^{(j)}(z): z \in \Sigma_{\delta}\right\}$ and $\left\{|z|^{j}\left(1+|z|^{-(2 q+2)}\right)^{-1}(B u)^{(j)}(z): z \in \Sigma_{\delta}\right\}$ are bounded in $E$.

Proof. Suppose first that $q=-1$ and $(\lambda B-A)^{-1} C A \subseteq A(\lambda B-A)^{-1} C$ $\left(\lambda \in \rho_{C}(A, B)\right)$; cf. (ii). Let $0<\delta^{\prime}<\delta<(\pi-\omega) / 2,1 / 2>\gamma_{0}>\delta /(\pi-\omega)$ and $\theta \in\left(\omega-\pi,(-\delta) / \gamma_{0}\right)$. Then, for every $\gamma \in\left(\gamma_{0}, 1 / 2\right)$, we have $\theta \in(\omega-\pi,(-\delta) / \gamma)$ and $\gamma>\delta /(\pi-\omega)$. Suppose that $\epsilon \in(0, \omega / 2)$ is sufficiently small. Define, for every $\gamma \in\left(\gamma_{0}, 1 / 2\right)$ and $x \in E$,

$$
\begin{aligned}
& F_{\gamma}(\lambda) x \\
& := \begin{cases}\frac{e^{i \theta \gamma} \sin \gamma \pi}{\pi} \int_{0}^{\infty} \frac{v^{\gamma}\left(v B+e^{i \theta} A\right)^{-1} C x d v}{\left(\lambda e^{i \theta \gamma}+v^{\gamma} \cos \pi \gamma\right)^{2}+v^{2 \gamma} \sin ^{2} \gamma \pi}, & \text { if } \arg (\lambda) \in(-\epsilon,(\pi / 2)+\delta), \\
\frac{e^{-i \theta \gamma} \sin \gamma \pi}{\pi} \int_{0}^{\infty} \frac{v^{\gamma}\left(v B+e^{-i \theta} A\right)^{-1} C x d v}{\left(\lambda e^{-i \theta \gamma}+v^{\gamma} \cos \pi \gamma\right)^{2}+v^{2 \gamma} \sin ^{2} \gamma \pi}, & \text { if } \arg (\lambda) \in(-(\pi / 2)-\delta, \epsilon) .\end{cases}
\end{aligned}
$$

If $x \in E$ and $\arg (\lambda) \in(-\epsilon,(\pi / 2)+\delta)$, resp., $\arg (\lambda) \in(-(\pi / 2)-\delta, \epsilon)$, then it is very simple to prove that

$$
\int_{0}^{\infty} e^{-\lambda e^{i \theta \gamma} t} W_{\theta, \gamma}(t) x d t=\frac{\sin \gamma \pi}{\pi} \int_{0}^{\infty} \frac{v^{\gamma}\left(v B+e^{i \theta} A\right)^{-1} C x}{\left(\lambda e^{i \theta \gamma}+v^{\gamma} \cos \pi \gamma\right)^{2}+v^{2 \gamma} \sin ^{2} \gamma \pi} d v
$$

resp.,

$$
\int_{0}^{\infty} e^{-\lambda e^{-i \theta \gamma} t} W_{-\theta, \gamma}(t) x d t=\frac{\sin \gamma \pi}{\pi} \int_{0}^{\infty} \frac{v^{\gamma}\left(v B+e^{-i \theta} A\right)^{-1} C x}{\left(\lambda e^{-i \theta \gamma}+v^{\gamma} \cos \pi \gamma\right)^{2}+v^{2 \gamma} \sin ^{2} \gamma \pi} d v
$$

where we use the notation from the proof of Theorem 2.4. Define $\Gamma_{\vartheta}:=$ $\left\{t e^{i \vartheta}: t \geq 0\right\} \quad(\vartheta \in(-\pi, \pi])$. Since, for every $x \in E$, 


$$
\begin{aligned}
e^{i \theta \gamma} \int_{0}^{\infty} e^{-\lambda e^{i \theta \gamma} t} W_{\theta, \gamma}(t) x d t & =\int_{\Gamma_{\theta \gamma}} e^{-\lambda v} W_{\theta, \gamma}\left(v e^{-i \theta \gamma}\right) x d v \\
& =\int_{\Gamma_{\theta \gamma}} e^{-\lambda v} W_{\gamma}(v) x d v
\end{aligned}
$$

and

$$
\begin{aligned}
e^{-i \theta \gamma} \int_{0}^{\infty} e^{-\lambda e^{-i \theta \gamma} t} W_{-\theta, \gamma}(t) x d t & =\int_{\Gamma_{-\theta \gamma}} e^{-\lambda v} W_{-\theta, \gamma}\left(v e^{i \theta \gamma}\right) x d v \\
& =\int_{\Gamma_{-\theta \gamma}} e^{-\lambda v} W_{\gamma}(v) x d v
\end{aligned}
$$

(cf. the construction of $\left(W_{\gamma}(z)\right)_{z \in \Sigma_{(\pi / 2)-\omega \gamma}}$, and observe that $\left.|\theta \gamma| \in(\delta, \gamma(\pi-\omega))\right)$, the Cauchy formula yields that

$$
e^{i \theta \gamma} \int_{0}^{\infty} e^{-\lambda e^{i \theta \gamma} t} W_{\theta, \gamma}(t) x d t=e^{-i \theta \gamma} \int_{0}^{\infty} e^{-\lambda e^{-i \theta \gamma} t} W_{-\theta, \gamma}(t) x d t, \quad \lambda \in \Sigma_{\epsilon}
$$

This, in turn, implies that the function $\lambda \mapsto F_{\gamma}(\lambda) x, \lambda \in \Sigma_{(\pi / 2)+\delta}$ is well defined, analytic and bounded by Const $\delta_{\delta^{\prime}}|\lambda|^{-1}$ on $\Sigma_{(\pi / 2)+\delta^{\prime}}(x \in E)$. Speaking-matter-offactly, it is not difficult to prove with the help of [22, Theorem 3.4] (cf. also [1, Theorem 2.6.1] for the Banach space case) and the uniqueness theorem for the Laplace transform that

$$
W_{\gamma}(z) x=\frac{1}{2 \pi i} \int_{\Gamma_{\delta^{\prime}, z}} e^{\lambda z} F_{\gamma}(\lambda) x d \lambda, \quad x \in E, z \in \Sigma_{\delta^{\prime}}, \gamma \in\left(\gamma_{0}, 1 / 2\right),
$$

where $\quad \Gamma_{\delta^{\prime}, z}:=\Gamma_{\delta^{\prime}, z, 1} \cup \Gamma_{\delta^{\prime}, z, 2}, \quad \Gamma_{\delta^{\prime}, z, 1}:=\left\{r e^{i\left((\pi / 2)+\delta^{\prime}\right)}: r \geq|z|^{-1}\right\} \cup\left\{|z|^{-1} e^{i \vartheta}:\right.$ $\left.\vartheta \in\left[0,(\pi / 2)+\delta^{\prime}\right]\right\} \quad$ and $\quad \Gamma_{\delta^{\prime}, z, 2}:=\left\{r e^{-i\left((\pi / 2)+\delta^{\prime}\right)}: r \geq|z|^{-1}\right\} \cup\left\{|z|^{-1} e^{i \vartheta}: \vartheta \in\right.$ $\left.\left[-(\pi / 2)-\delta^{\prime}, 0\right]\right\}$ are oriented counterclockwise. Applying the dominated convergence theorem, we get that

$$
\begin{aligned}
\lim _{\gamma \rightarrow 1 / 2-} W_{\gamma}(z) x= & \frac{e^{i \theta / 2}}{2 \pi^{2} i} \int_{\Gamma_{\delta^{\prime}, z, 1}} e^{\lambda z} \int_{0}^{\infty} \frac{v^{1 / 2}\left(v B+e^{i \theta} A\right)^{-1} C x}{\lambda^{2} e^{i \theta}+v} d v d \lambda \\
& +\frac{e^{-i \theta / 2}}{2 \pi^{2} i} \int_{\Gamma_{\delta^{\prime}, z, 2}} e^{\lambda z} \int_{0}^{\infty} \frac{v^{1 / 2}\left(v B+e^{-i \theta} A\right)^{-1} C x}{\lambda^{2} e^{-i \theta}+v} d v d \lambda \\
:= & W_{1 / 2}(z) x, \quad x \in E, z \in \Sigma_{\delta^{\prime}} .
\end{aligned}
$$

Define $F_{1 / 2}(\lambda)$ by replacing the number $\gamma$ with the number $1 / 2$ in definition of $W_{\gamma}(\lambda)$. Then, for every $x \in E$, the function $\lambda \mapsto F_{1 / 2}(\lambda) x, \lambda \in \Sigma_{(\pi / 2)+\delta}$ is well 
defined and analytic on $\Sigma_{(\pi / 2)+\delta}$ because $F_{1 / 2}(\lambda) x=\lim _{\gamma \rightarrow 1 / 2-} F_{\gamma}(\lambda) x, \lambda \in \Sigma_{(\pi / 2)+\delta}$ and the convergence is uniform on compacts of $\Sigma_{(\pi / 2)+\delta}$ (cf. [22, Lemma 3.3]). Furthermore, we can argue as in the proof of estimate [21, (334)] so as to conclude that for each $q \in \circledast$ there exists $r_{q} \in \circledast$ such that $q\left(F_{1 / 2}(\lambda) x\right) \leq r_{q}(x)$ Const $_{\delta^{\prime}}|\lambda|^{-1}$, $\lambda \in \Sigma_{(\pi / 2)+\delta^{\prime}}, x \in E$. Now it becomes apparent that we can define the operator family $\left(W_{1 / 2}(z)\right)_{z \in \Sigma_{(\pi-\omega) / 2}} \subseteq L(E)$ by $W_{1 / 2}(z) x:=\lim _{\gamma \rightarrow 1 / 2-} W_{\gamma}(z) x, \quad z \in \Sigma_{(\pi-\omega) / 2}$, $x \in E$; this operator family is equicontinuous on any proper subsector of $\Sigma_{(\pi-\omega) / 2}$ and satisfies additionally that the mapping $z \mapsto W_{1 / 2}(z) x, \quad z \in \Sigma_{(\pi-\omega) / 2}$ is analytic for all $x \in E$. Let us prove that for each $x \in \overline{D(A)}$ the function $u(z):=W_{1 / 2}(z) x, \quad z \in \Sigma_{(\pi-\omega) / 2}$ is a solution of problem $\left(P_{2, q, B}\right)$ with $q=-1$. Suppose first that $x \in D(A)$. Then the condition $(\lambda B-A)^{-1} C A \subseteq A(\lambda B-A)^{-1} C$ $\left(\lambda \in \rho_{C}(A, B)\right)$ in combination with the closedness of $A$ shows that $W_{1 / 2}(z) A x=$ $A W_{1 / 2}(z) x, z \in \Sigma_{(\pi-\omega) / 2}$. By the foregoing, we also have that the operator family $\left(B W_{1 / 2}(z)\right)_{z \in \Sigma_{(\pi-\omega) / 2}} \subseteq L(E)$ is equicontinuous and the mapping $z \mapsto$ $B W_{1 / 2}(z) x, z \in \Sigma_{(\pi-\omega) / 2}$ is analytic $(x \in E)$, as well as that $\left(d^{2} / d z^{2}\right) B W_{1 / 2}(z) x=$ $B\left(d^{2} / d z^{2}\right) W_{1 / 2}(z) x, \quad z \in \Sigma_{(\pi-\omega) / 2}, \quad x \in E$ and $B W_{1 / 2}(z) \in L(E),\left(d^{2} / d z^{2}\right) B W_{1 / 2}(z)$ $\in L(E), B\left(d^{2} / d z^{2}\right) W_{1 / 2}(z) \in L(E)$ for $z \in \Sigma_{(\pi-\omega) / 2}$. Furthermore, the dominated convergence theorem yields that

$$
\lim _{\gamma \rightarrow 1 / 2-} B W_{\gamma}^{(n)}(z)=B W_{1 / 2}^{(n)}(z) x, \quad z \in \Sigma_{(\pi-\omega) / 2}, x \in E, n \in \mathbf{N}_{0} .
$$

By Theorem 2.5(ii) and definition of modified Liouville right-sided fractional derivatives, we get that

$$
\frac{d^{2}}{d s^{2}} \int_{0}^{\infty} g_{3-1 / \gamma}(t) B W_{\gamma}^{\prime}(t+s) x d t=-W_{\gamma}(s) A x, \quad s>0, \gamma \in\left(\gamma_{0}, 1 / 2\right),
$$

i.e.,

$$
\int_{0}^{\infty} g_{3-1 / \gamma}(t) B W_{\gamma}^{\prime \prime \prime}(t+s) x d t=-W_{\gamma}(s) A x, \quad s>0, \gamma \in\left(\gamma_{0}, 1 / 2\right) .
$$

The integration by parts leads us to the following

$$
-\int_{0}^{\infty} g_{4-1 / \gamma}(t) B W_{\gamma}^{(i v)}(t+s) x d t=-W_{\gamma}(s) A x, \quad s>0, \gamma \in\left(\gamma_{0}, 1 / 2\right) .
$$

Using again the dominated convergence theorem, we obtain by letting $\gamma \rightarrow 1 / 2-$ that

$$
\int_{0}^{\infty} t B W_{1 / 2}^{(i v)}(t+s) x d s=W_{1 / 2}(s) A x, \quad s>0
$$


which clearly implies after an application of the partial integration that $B W_{1 / 2}^{\prime \prime}(s)=A W_{1 / 2}(s) x, s>0$. By the uniqueness theorem for analytic functions, this equality continues to hold for all $z \in \Sigma_{(\pi-\omega) / 2}$. The foregoing arguments also imply that we can compute $F_{\gamma}(\lambda) x$ for $\lambda>0\left(\gamma \in\left(\gamma_{0}, 1 / 2\right)\right)$ by plugging $\theta=0$ in any of two terms appearing in (2.8). Taking the limit as $\gamma \rightarrow 1 / 2-$, we get that $F_{1 / 2}(\lambda) x=\pi^{-1} \int_{0}^{\infty} v^{1 / 2}\left(\lambda^{2}+v\right)^{-1}(v B+A)^{-1} C x d v, \lambda>0$. Now we will prove that $B W_{1 / 2}(z) x-C x \rightarrow 0$ as $z \rightarrow 0, z \in \Sigma_{(\pi / 2)+\delta^{\prime}}$. Due to [22, Theorem 3.4], it suffices to show that $\lim _{\lambda \rightarrow+\infty} \lambda B F_{1 / 2}(\lambda) x=C x$. This follows by applying the dominated convergence theorem on the integral appearing on the right-hand side of the equality

$$
\lambda B F_{1 / 2}(\lambda) x-C x=\frac{1}{\pi} \int_{0}^{\infty} \frac{v^{1 / 2} \lambda}{\lambda^{2}+v}\left[B(v B+A)^{-1} C x-\frac{C x}{v}\right] d v
$$

and by observing that

$$
q\left(B(v B+A)^{-1} C x-\frac{C x}{v}\right)=\frac{1}{v} q\left((v B+A)^{-1} C A x\right)=O\left(v^{-2}\right), \quad q \in \circledast, v>1
$$

and

$$
q\left(B(v B+A)^{-1} C x-\frac{C x}{v}\right)=O\left(v^{-1}\right), \quad q \in \circledast, v \in(0,1) .
$$

Keeping in mind the Cauchy integral formula, the proof of (ii) follows immediately in the case that $q=-1$ and $x \in D(A)$. The proof of (ii) in the case that $q=-1$ and $x \in \overline{D(A)}$ follows from the standard limit procedure. If $(-3) / 2<q<-1$, then for each $\varepsilon>0$ the condition $(\mathrm{H})$, with the operators $A$ and $B$ replaced respectively by $A+\varepsilon B$ and $B$, holds with $q=-1$ and the same spectral angle $\omega$; in this case, the proof of (ii) can be deduced by slightly modifying the corresponding part of the proof of [21, Theorem 2.9.51(iii.2)]. The proof of (i) is very similar to that of (ii); for the sake of completeness, we will include almost all relevant details. As in the proof of (ii), it will be assumed that $0<\delta^{\prime}<\delta<(\pi-\omega) / 2,1 / 2>\gamma_{0}>\delta /(\pi-\omega), \theta \in\left(\omega-\pi,(-\delta) / \gamma_{0}\right)$ and that $\epsilon \in(0, \omega / 2)$ is sufficiently small. Due to the proof of [21, Theorem 2.9.48] (cf. also the estimate (2.4)), we have that, for every $\theta^{\prime} \in(\omega-\pi, \pi-\omega)$ and $\gamma \in(q+1,1 / 2)$, the mapping $t \mapsto W_{\theta^{\prime}, \gamma}(t) x, t \geq 0(x \in E)$ satisfies the condition $(\mathrm{P} 1)$, as well as that

$$
\mathscr{L}\left(W_{\theta^{\prime}, \gamma}(t) x\right)(\mu)=\frac{\sin \gamma \pi}{\pi} \int_{0}^{\infty} \frac{v^{\gamma}\left(v B+e^{i \theta^{\prime}} A\right)^{-1} C x}{\left(\mu+v^{\gamma} \cos \pi \gamma\right)^{2}+v^{2 \gamma} \sin ^{2} \gamma \pi} d v, \quad \mu \in \mathbf{C}_{+}, x \in E,
$$


and that for each $q \in \circledast$ there exists $r_{q} \in \circledast$ such that

$$
\begin{aligned}
& q\left(\mathscr{L}\left(W_{\theta^{\prime}, \gamma}(t) x\right)(\mu)\right) \\
& \quad=r_{q}(x) O\left(|\mu|^{-1}+|\mu|^{(q+1) / \gamma-1}\right), \quad \mu \in \Sigma_{\pi / 2-\varepsilon}, x \in E(\varepsilon \in(0, \pi / 2)) .
\end{aligned}
$$

This implies that, for every $\gamma \in\left(\max \left(\gamma_{0}, q+1\right), 1 / 2\right)$ and $x \in E$, we can define $F_{\gamma}(\lambda) x$ through (2.8). If $x \in E$ and $\arg (\lambda) \in(-\epsilon,(\pi / 2)+\delta)$, resp., $\arg (\lambda) \epsilon$ $(-(\pi / 2)-\delta, \epsilon)$, then the equality (2.9), resp., (2.10), continues to hold for those values of parameter $\gamma$. Furthermore, (2.11) holds for any $\lambda \in \Sigma_{\epsilon}$; hence, the function $\lambda \mapsto F_{\gamma}(\lambda) x, \lambda \in \Sigma_{(\pi / 2)+\delta}$ is well defined, analytic and bounded by Const $_{\delta^{\prime}}\left(|\lambda|^{-1}+|\lambda|^{((q+1) / \gamma)-1}\right)$ on $\Sigma_{(\pi / 2)+\delta^{\prime}} \quad\left(x \in E, \quad \gamma \in\left(\max \left(\gamma_{0}, q+1\right), 1 / 2\right)\right)$. If $x \in E, z \in \Sigma_{\delta^{\prime}}, \gamma \in\left(\max \left(\gamma_{0}, q+1\right), 1 / 2\right)$ and $\zeta \geq 0$, then we define

$$
W_{\gamma}^{(-\zeta)}(z) x:=\frac{1}{2 \pi i} \int_{\Gamma_{\delta^{\prime}, z}^{\omega^{\prime}}} e^{\lambda z} \lambda^{-\zeta} F_{\gamma}(\lambda) x d \lambda,
$$

where $\omega^{\prime}>0$ is taken arbitrarily, $\quad \Gamma_{\delta^{\prime}, z}^{\omega^{\prime}}:=\Gamma_{\delta^{\prime}, z, 1}^{\omega^{\prime}} \cup \Gamma_{\delta^{\prime}, z, 2}^{\omega^{\prime}}, \quad \Gamma_{\delta^{\prime}, z, 1}^{\omega^{\prime}}:=\left\{\omega^{\prime}+\right.$ $\left.r e^{i\left((\pi / 2)+\delta^{\prime}\right)}: r \geq|z|^{-1}\right\} \cup\left\{\omega^{\prime}+|z|^{-1} e^{i \vartheta}: \vartheta \in\left[0,(\pi / 2)+\delta^{\prime}\right]\right\} \quad$ and $\quad \Gamma_{\delta^{\prime}, z, 2}^{\omega^{\prime}}:=\left\{\omega^{\prime}+\right.$ $\left.r e^{-i\left((\pi / 2)+\delta^{\prime}\right)}: r \geq|z|^{-1}\right\} \cup\left\{\omega^{\prime}+|z|^{-1} e^{i \vartheta}: \vartheta \in\left[-(\pi / 2)-\delta^{\prime}, 0\right]\right\}$ are oriented counterclockwise. By [22, Theorem 3.4], we get that $\mathscr{L}\left(W_{\gamma}^{(-1)}(t) x\right)(\lambda)=\lambda^{-1} F_{\gamma}(\lambda) x$, $\lambda>0, x \in E$; using this fact, as well as the equality $\mathscr{L}\left(W_{\gamma}(t) x\right)(\lambda)=F_{\gamma}(\lambda) x$, $\lambda>0, x \in E$, the uniqueness theorem for Laplace transform and the uniqueness theorem for analytic functions, we obtain that $(d / d z) W_{\gamma}^{(-1)}(z) x=W_{\gamma}(z) x, x \in E$, $z \in \Sigma_{\delta^{\prime}}\left(\gamma \in\left(\max \left(\gamma_{0}, q+1\right), 1 / 2\right)\right)$. On the other hand, the dominated convergence theorem yields that $(d / d z) W_{\gamma}^{(-1)}(z) x=W_{\gamma}^{(0)}(z) x$, so that $W_{\gamma}^{(0)}(z) x=$ $W_{\gamma}(z) x, x \in E, z \in \Sigma_{\delta^{\prime}}\left(\gamma \in\left(\max \left(\gamma_{0}, q+1\right), 1 / 2\right)\right)$. Define $F_{1 / 2}(\lambda)\left(\lambda \in \Sigma_{(\pi / 2)+\delta}\right)$ and $\left(W_{1 / 2}(z)\right)_{z \in \Sigma_{\delta^{\prime}}} \subseteq L(E)$ in exactly the same way as in the proof of (ii). As before, we have that, for every $x \in E$, the function $\lambda \mapsto F_{1 / 2}(\lambda) x, \lambda \in \Sigma_{(\pi / 2)+\delta}$ is well defined and analytic on $\Sigma_{(\pi / 2)+\delta}$ because $F_{1 / 2}(\lambda) x=\lim _{\gamma \rightarrow 1 / 2-} F_{\gamma}(\lambda) x, \lambda \in \Sigma_{(\pi / 2)+\delta}$ and the convergence is uniform on compacts of $\Sigma_{(\pi / 2)+\delta}$; furthermore, for each $q \in \circledast$ there exists $r_{q} \in \circledast$ such that $q\left(F_{1 / 2}(\lambda) x\right) \leq r_{q}(x)$ Const $_{\delta^{\prime}}\left(|\lambda|^{-1}+|\lambda|^{2 q+1}\right)$, $\lambda \in \Sigma_{(\pi / 2)+\delta^{\prime}}, x \in E$. Then the limit equality (2.12) continues to hold (with the replacements of contours $\Gamma_{\delta^{\prime}, z, 1}, \quad \Gamma_{\delta^{\prime}, z, 2}$ with $\Gamma_{\delta^{\prime}, z, 1}^{\omega^{\prime}}, \quad \Gamma_{\delta^{\prime}, z, 2}^{\omega^{\prime}}$, respectively), the operator families $\left(W_{1 / 2}(z)\right)_{z \in \Sigma_{(\pi-\omega) / 2}} \subseteq L(E)$ and $\left(B W_{1 / 2}(z)\right)_{z \in \Sigma_{(\pi-\omega) / 2}} \subseteq L(E)$ can be defined in the very obvious way, and we have that $\lim _{\gamma \rightarrow 1 / 2-} B W_{\gamma}^{(n)}(z) x=$ $B W_{1 / 2}^{(n)}(z) x$ for all $x \in E, z \in \Sigma_{(\pi-\omega) / 2}$ and $n \in \mathbf{N}_{0}$. Arguing as in the proof of [21, Theorem 2.9.51(iii.1)], we can show that, for every $j \in \mathbf{N}_{0}$, the families $\left\{|z|^{j}(1+\right.$ $\left.\left.|z|^{-(2 q+2)}\right)^{-1} W_{1 / 2}^{(j)}(z): z \in \Sigma_{\delta^{\prime}}\right\} \subseteq L(E) \quad$ and $\quad\left\{|z|^{j}\left(1+|z|^{-(2 q+2)}\right)^{-1}\left(B W_{1 / 2}\right)^{(j)}(z)\right.$ : 
$\left.z \in \Sigma_{\delta^{\prime}}\right\} \subseteq L(E)$ are equicontinuous. Suppose now that $x \in D(A)$. Then $A W_{1 / 2}(z) x$ $=W_{1 / 2}(z) A x, \quad z \in \Sigma_{(\pi-\omega) / 2}$ and for each $q \in \circledast$ there exist $c_{q, \delta^{\prime}}>0$ and $r_{q} \in \circledast$ such that (cf. (2.14)-(2.15) with $\theta=0$ ):

$$
\begin{aligned}
& q\left(\frac{e^{i \theta / 2}}{\pi} \int_{0}^{\infty} \frac{v^{1 / 2} B\left(v B+e^{i \theta} A\right)^{-1} C x}{\lambda^{2} e^{i \theta}+v} d v-\frac{C x}{\lambda}\right) \\
& \quad \leq c_{q, \delta^{\prime}}\left[r_{q}(x)+r_{q}(A x)\right]\left[\int_{0}^{1} \frac{v^{1 / 2}}{|\lambda|^{2}+v}\left(\frac{1}{v}+v^{q}\right) d v+\int_{1}^{\infty} \frac{v^{1 / 2}}{|\lambda|^{2}+v} \frac{1}{v}\left(\frac{1}{v}+v^{q}\right) d v\right] \\
& \quad \leq c_{q, \delta^{\prime}}\left[r_{q}(x)+r_{q}(A x)\right]|\lambda|^{-1}, \quad \arg (\lambda) \in\left(-\epsilon,(\pi / 2)+\delta^{\prime}\right) .
\end{aligned}
$$

If $\arg (\lambda) \in\left(-\left((\pi / 2)+\delta^{\prime}\right),-\epsilon\right)$, then we can estimate the term

$$
q\left(\frac{e^{-i \theta / 2}}{\pi} \int_{0}^{\infty} \frac{v^{1 / 2} B\left(v B+e^{-i \theta} A\right)^{-1} C x}{\lambda^{2} e^{-i \theta}+v} d v-\frac{C x}{\lambda}\right)
$$

in the same way as above, from which we may conclude that $q\left(B F_{1 / 2}(\lambda) A x\right)=$ $\left(r_{q}(x)+r_{q}(A x)\right) O\left(|\lambda|^{-1}\right), \lambda \in \Sigma_{(\pi / 2)+\delta^{\prime}}$. A similar line of reasoning as performed in the case that $q=-1$ and $x \in D(A)$ enables us to deduce that

$$
\lambda\left[\frac{e^{i \theta / 2}}{\pi} \int_{0}^{\infty} \frac{v^{1 / 2} B\left(v B+e^{i \theta} A\right)^{-1} C x}{\lambda^{2} e^{i \theta}+v} d v-\frac{C x}{\lambda}\right] \rightarrow 0, \quad \lambda \rightarrow+\infty .
$$

Applying [22, Theorem 3.4] and (2.16), we get that $\mathscr{L}\left(B W_{1 / 2}(t) x\right)(\lambda)=B F_{1 / 2}(\lambda) x$, $\lambda>0$ and $\lim _{z \rightarrow 0, z \in \Sigma_{\delta^{\prime}}} B W_{1 / 2}(z) x=C x$. In the proof of Theorem 2.5, we have proved that $B \frac{d^{j}}{d z^{j}} W_{\gamma}(z) x=\frac{d^{j}}{d z^{j}} B W_{\gamma}(z) x\left(z \in \Sigma_{\pi / 2-\gamma \pi}, j \in \mathbf{N}\right)$. As a consequence of this equality and the Cauchy integral formula, we have that $q\left(B \frac{d^{j}}{d t^{j}} W_{\gamma}(t) x\right)=$ $O\left(t^{-j}\right), t>0$. On the other hand, the proof of [21, Theorem 2.9.48] shows that the equation (2.13) continues to hold, which simply implies that $B W_{1 / 2}^{\prime \prime}(z) x=$ $A W_{1 / 2}(z) x, z \in \Sigma_{(\pi-\omega) / 2}$. This completes the proof of theorem.

The main problem in application of Theorem 2.5-Theorem 2.6 to incomplete abstract degenerate differential equations lies in the fact that, in many concrete situation, any of conditions $(\lambda B-A)^{-1} C A \subseteq A(\lambda B-A)^{-1} C\left(\lambda \in \rho_{C}(A, B)\right)$ and $(\lambda B-A)^{-1} C B \subseteq B(\lambda B-A)^{-1} C\left(\lambda \in \rho_{C}(A, B)\right)$ is not satisfied. Suppose, for example, that $\varnothing \neq \Omega \subseteq \mathbf{R}^{n}$ is an open bounded set with $C^{\infty}$-boundary, $E:=L^{2}(\Omega)$, $A:=\Delta$ with the Dirichlet boundary conditions, $a(x) \in L^{\infty}(\Omega), a(x) \leq 0$ on $\bar{\Omega}$, $a(x)<0$ almost everywhere in $\Omega$, and $B f(x):=a(x)^{-1} f(x)$ with maximal domain 
(cf. [13, Example 3.8, pp. 81-83]). Then $B^{-1} \in L(E)$ and the operator $A B^{-1}$ is closed. Suppose, in addition, that $a^{-1} \in L^{r}(\Omega)$ for some $r \geq 2$ (resp., $r>2, r>n$ ), if $n=1$ (resp., $n=2, n \geq 3$ ). Then it has been proved in the above-mentioned example that the condition [21, (HQ), p. 207] holds with the number $\omega=0$, $C=I, m=-1+(n / 2 r) \in(-1,(-1 / 2))$, and with the operator $A$ replaced by $A B^{-1}$ therein. This implies by [21, Theorem 2.9.51(i.3)] that the operator $-\left(A B^{-1}\right)_{1 / 2}$ is the integral generator of an exponentially bounded, analytic $(n / r)$ times integrated semigroup of angle $\pi / 2$ on $E$, and that the abstract incomplete Cauchy problem $\left[21,\left(P_{2, m}\right)\right]$, which corresponds to the equation $u_{t t}(t, x)=$ $\Delta\{a(x) u(t, x)\}, t>0$, has a unique solution that is analytically extensible to the right half plane. It is clear that Theorem 2.6 cannot be applied here directly, by regarding the problem $u_{t t}(t, x)=\Delta\{a(x) u(t, x)\}, t>0$ as a problem of the form $\left(P_{2, q, B}\right)$ considered above.

Now we would like to illustrate the abstract theoretical results established in this paper with the following example.

Example 2.7. Assume that $n \in \mathbf{N}$ and $i A_{j}, 1 \leq j \leq n$ are commuting generators of bounded $C_{0}$-groups on a Banach space $E$; let us recall that $\mathbf{A}:=$ $\left(A_{1}, \ldots, A_{n}\right)$. Assume, further, that $0<\delta<2, P_{1}(x)$ and $P_{2}(x)$ are non-zero complex polynomials, $N_{1}=d g\left(P_{1}(x)\right), N_{2}=d g\left(P_{2}(x)\right), \beta>\frac{n}{2} \frac{\left(N_{1}+N_{2}\right)}{\min (1, \delta)}$ (resp. $\beta \geq$ $n\left|\frac{1}{p}-\frac{1}{2}\right| \frac{\left(N_{1}+N_{2}\right)}{\min (1, \delta)}$, if $E=L^{p}\left(\mathbf{R}^{n}\right)$ for some $\left.1<p<\infty\right), P_{2}(x) \neq 0, x \in \mathbf{R}^{n}$ and

$$
\sup _{x \in \mathbf{R}^{n}} \Re\left(\left(\frac{P_{1}(x)}{P_{2}(x)}\right)^{1 / \delta}\right) \leq 0 .
$$

Define

$$
\begin{aligned}
& R_{\delta}(t):=\left(E_{\delta}\left(t^{\delta} \frac{P_{1}(x)}{P_{2}(x)}\right)\left(1+|x|^{2}\right)^{-\beta / 2}\right)(\mathbf{A}), \quad t \geq 0, G_{\delta}(t):={\overline{P_{2}(\mathbf{A})}}^{-1} R_{\delta}(t), t \geq 0, \\
& A^{\prime}:=\overline{P_{1}(\mathbf{A})}, \quad B^{\prime}:=\overline{P_{2}(\mathbf{A})} \text { and } C:=R_{\delta}(0) . \text { Then we know that }\left\|R_{\delta}(t)\right\|+ \\
& \left\|G_{\delta}(t)\right\|=O\left(1+t^{\max (1, \delta) n / 2}\right), \quad t \geq 0 \quad\left(\left\|R_{\delta}(t)\right\|+\left\|G_{\delta}(t)\right\|=O\left(1+t^{\max (1, \delta) n|1 / p-1 / 2|}\right),\right. \\
& \left.t \geq 0, \text { if } E=L^{p}\left(\mathbf{R}^{n}\right) \text { for some } 1<p<\infty\right),
\end{aligned}
$$

$$
\lambda^{\delta-1}\left(\lambda^{\delta} B^{\prime}-A^{\prime}\right)^{-1} C x=\int_{0}^{\infty} e^{-\lambda t} G_{\delta}(t) x d t, \quad \Re \lambda>0, x \in E,
$$

and

$$
\lambda^{\delta-1} B^{\prime}\left(\lambda^{\delta} B^{\prime}-A^{\prime}\right)^{-1} C x=\int_{0}^{\infty} e^{-\lambda t} B^{\prime} G_{\delta}(t) x d t=\int_{0}^{\infty} e^{-\lambda t} R_{\delta}(t) x d t, \quad \Re \lambda>0, x \in E ;
$$


cf. [23]-[24] for more details. This implies

$$
\begin{aligned}
& \left\|\left(\lambda B^{\prime}-A^{\prime}\right)^{-1} C\right\|+\left\|B^{\prime}\left(\lambda B^{\prime}-A^{\prime}\right)^{-1} C\right\| \\
& \quad=O\left(|\lambda|^{-1}+|\lambda|^{-1-(\max (1, \delta) n) /(2 \delta)}\right), \quad \lambda \in \Sigma_{\delta \pi / 2}
\end{aligned}
$$

in the case of a general space $E$, and

$$
\begin{aligned}
& \left\|\left(\lambda B^{\prime}-A^{\prime}\right)^{-1} C\right\|+\left\|B^{\prime}\left(\lambda B^{\prime}-A^{\prime}\right)^{-1} C\right\| \\
& \quad=O\left(|\lambda|^{-1}+|\lambda|^{-1-(\max (1, \delta) n|1 / p-1 / 2|) / \delta}\right), \quad \lambda \in \Sigma_{\delta \pi / 2},
\end{aligned}
$$

in the case that $E=L^{p}\left(\mathbf{R}^{n}\right)$ for some $1<p<\infty$ (cf. also [23, Remark 4.4(ii)] and [23, Remark 4.5], which enables us to consider the wellposedness of related degenerate Cauchy problems in $E_{l}$-type spaces [42]). Setting $A:=-A^{\prime}$ and $B:=B^{\prime}$, we have that the condition $(\mathrm{H})$ holds wih $\omega=\pi-(\delta \pi / 2)$ as well as that $C^{-1} A C=A, C^{-1} B C=B,(\lambda B-A)^{-1} C A \subseteq A(\lambda B-A)^{-1} C$ and $(\lambda B-A)^{-1} C B \subseteq$ $B(\lambda B-A)^{-1} C\left(\lambda \in \rho_{C}(A, B)\right)$, which implies that Theorem 2.5(ii), resp., Theorem 2.6(ii) is susceptible to applications in the case that $E=L^{p}\left(\mathbf{R}^{n}\right)$ for some $1<p<\infty$ satisfying

$$
\frac{\max (1, \delta) n\left|\frac{1}{p}-\frac{1}{2}\right|}{\delta}<\gamma<1 / 2, \quad \text { resp. }, \quad \frac{\max (1, \delta) n\left|\frac{1}{p}-\frac{1}{2}\right|}{\delta}<1 / 2 .
$$

Before proceeding further, we would like to mention in passing that the operator $B$ is injective (cf. [23, Remark 4.4(i)]) and that the operator $B^{-1} A$ is closable because $\rho_{C^{\prime}}\left(B^{-1} A\right) \neq \varnothing \quad\left(C^{\prime}:=C(B+A)^{-1} C\right)$, which follows from the equality $\left(\lambda+B^{-1} A\right)^{-1} C(B+A)^{-1} C x=B(\lambda B+A)^{-1} C(B+A)^{-1} C x, \quad \lambda>0$, $x \in E$ (cf. (2.6)). It is also worth noting that the operator $A B^{-1}$ is closable because $\rho_{C}\left(A B^{-1}\right) \neq \varnothing$; this is a consequence of the equality $\left(\lambda+A B^{-1}\right)^{-1} C x=$ $B(\lambda B+A)^{-1} C x, \lambda>0, x \in E$. Now we will prove the uniqueness of solution of problem $\left(P_{2, q, B}\right)$ in our particular case; recall that $E=L^{p}\left(\mathbf{R}^{n}\right)$ for some $1<p<\infty$ satisfying $\zeta:=\frac{\max (1, \delta) n\left|\frac{1}{p}-\frac{1}{2}\right|}{\delta}<1 / 2$. Denote by $\mathscr{M}_{C, q, \omega}$ the class which consists of all closed linear operators $D$ acting on $E$ such that $\mathbf{C} \backslash \overline{\Sigma_{\omega}} \subseteq \rho_{C}(D)$, $D C \subseteq C D$ and the family

$$
\left\{\left(|\lambda|^{-1}+|\lambda|^{q}\right)^{-1}(\lambda-D)^{-1} C: \lambda \notin \Sigma_{\omega^{\prime}}\right\}
$$

is equicontinuous for every $\omega<\omega^{\prime}<\pi$; here $q \in \mathbf{R}$ and $\omega \in[0, \pi)$. Then we have $\zeta \in[0,1 / 2)$ and our previous considerations show that $\overline{A B^{-1}} \in \mathscr{M}_{C,-1-\zeta, \pi-(\delta \pi / 2)}$. 
If $z \mapsto u(z), \quad z \in \Sigma_{(\pi-\omega) / 2}$ is a solution of problem $\left(P_{2, q, B}\right)$ with $x=0$, then $v(z):=B u(z), \quad z \in \Sigma_{(\pi-\omega) / 2}$ is a solution of problem

$$
\left(P_{2, q}\right):\left\{\begin{array}{l}
v \in \mathscr{A}\left(\Sigma_{(\pi / 2)-(\omega / 2)}: E\right), \\
\frac{d^{2}}{d z^{2}} v(z)=\overline{A B^{-1}} v(z), \quad z \in \Sigma_{(\pi / 2)-(\omega / 2)}, \\
\lim _{z \rightarrow 0, z \in \Sigma_{\delta}} v(z)=0, \quad \text { for every } \delta \in(0,(\pi / 2)-(\omega / 2)), \\
\text { the set }\left\{\left(1+|z|^{-(2 q+2)}\right)^{-1} v(z): z \in \Sigma_{\delta}\right\} \text { is bounded in } E \\
\text { for every } \delta \in(0,(\pi / 2)-(\omega / 2)) .
\end{array}\right.
$$

An application of [21, Theorem 2.9.51(iii.2)] yields $v(z)=0, z \in \Sigma_{(\pi-\omega) / 2}$, so that $u(z)=0, z \in \Sigma_{(\pi-\omega) / 2}$ by the injectiveness of $B$.

The analysis contained in Example 2.7 shows that there exists at most one solution of problem $\left(P_{2, q, B}\right)$ stated in Theorem 2.6(i), resp., Theorem 2.6(ii), provided that the operator $B$ is injective, the operator $A B^{-1}$ is closable and $\overline{A B^{-1}} \in \mathscr{M}_{C, q, \omega}$ for some $q \in(-1,(-1) / 2)$ and $\omega \in[0, \pi)$, resp., $q \in((-3 / 2),-1]$ and $\omega \in[0, \pi)$. Finally, we would like to raise the following issue: In which other cases the uniqueness of solutions of problem $\left(P_{2, q, B}\right)$ can be proved $(B \neq I)$ ?

\section{References}

[1] Arendt, W., Batty, C. J. K., Hieber, M., and Neubrander, F., Vector-valued Laplace Transforms and Cauchy Problems, Birkhäuser Verlag, 2001.

[2 2 Arendt, W. and Favini, A., Integrated solutions to implicit differential equations, Rend. Sem. Mat. Univ. Poi. Torino 51 (1993), 315-329.

[3] Balakrishnan, A. V., Fractional powers of closed operators and the semigroups generated by them, Pacific J. Math. 10 (1960), 419-437.

[4] Bazhlekova, E., Fractional Evolution Equations in Banach Spaces, PhD Thesis, Eindhoven University of Technology, Eindhoven, 2001.

[ 5 ] Carroll, R. W. and Showalter, R. W., Singular and Degenerate Cauchy Problems, Academic Press, New York, 1976.

[ 6 ] Chen, C., Kostić, M., Li, M., and Žigić, M., Complex powers of $C$-sectorial operators. Part I, Taiwanese J. Math. 17 (2013), 465-499.

[7] Chen, C., Kostić, M., and Li, M., Complex powers of almost $C$-nonnegative operators, Contemp. Anal. Appl. Math. 2 (2014), 1-77.

[ 8 ] Da Prato, G., Semigruppi regolarizzabilli, Ricerche Mat. 15 (1966), 223-248.

[9] deLaubenfels, R., Existence Families, Functional Calculi and Evolution Equations, Lecture Notes in Mathematics 1570, Springer-Verlag, New York, 1994.

[10] Demidenko, G. V. and Uspenskii, S. V., Partial Differential Equations And Systems Not Solvable With Respect To The Highest-Order Derivative, Vol. 256 of Pure and Applied Mathematics Series, CRC Press, New York, 2003.

[11] Diethelm, K., The Analysis of Fractional Differential Equations, Springer-Verlag, Berlin, 2010.

[12] Favaron, A. and Favini, A., Fractional powers and interpolation theory for multivalued linear operators and applications to degenerate differential equations, Tsukuba J. Math. 35 (2011), 259-323. 
[13] Favini, A. and Yagi, A., Degenerate Differential Equations in Banach Spaces, Chapman and Hall/CRC Pure and Applied Mathematics, New York, 1998.

[14] Favini, A. and Yagi, A., Multivalued linear operators and degenerate evolution equations, Ann. Mat. Pura Appl. 163 (1993), 353-384.

[15] Favini, A., An operational method for abstract degenerate evolution equations of hyperbolic type, J. Funct. Anal. 76 (1988), 432-456.

[16] Fedorov, V. E., A class of second-order equations of Sobolev type and degenerate groups of operators, Vestn. Chelyab. Gos. Univ. Mat. Mekh. Inform. 26(13) (2011), 59-75.

[17] Fedorov, V. E. and Debbouche, A., A class of degenerate fractional evolution systems in Banach spaces, Differ. Uravn. 49 (2013), 1569-1576.

[18] Fedorov, V. E. and Gordievskikh, D. M., Resolving operators of degenerate evolution equations with fractional derivative with respect to time, Russian Math. 1 (2015), 71-83.

[19] Kilbas, A. A., Srivastava, H. M., and Trujillo, J. J., Theory and Applications of Fractional Differential Equations, Elsevier Science B.V., Amsterdam, 2006.

[20] Kostić, M., Generalized Semigroups and Cosine Functions, Mathematical Institute SANU, Belgrade, 2011.

[21] Kostić, M., Abstract Volterra Integro-Differential Equations, Taylor and Francis Group/CRC Press/Science Publishers, Boca Raton, Florida, 2015.

[22] Kostić, M., Abstract Volterra equations in locally convex spaces, Sci. China Math. 55 (2012), 1797-1825.

[23] Kostić, M., Degenerate abstract Volterra equations in locally convex spaces, Filomat, in press.

[24] Kostić, M., Degenerate multi-term fractional differential equations in locally convex spaces, Publ. Inst. Math., Nouv. Sér., in press.

[25] Kostić, M., Hypercyclic and topologically mixing properties of degenerate multi-term fractional differential equations, Differ. Equ. Dyn. Syst., DOI: 10.1007/s12591-015-0238-x, in press.

[26] Kostić, M., $\mathscr{D}$-Hypercyclic and $\mathscr{D}$-topologically mixing properties of degenerate multi-term fractional differential equations, Azerbaijan J. Math. 5 (2015), 77-94.

[27] Kostić, M., Entire and analytical solutions of abstract degenerate differential equations and their systems, preprint.

[28] Kostić, M., Degenerate $k$-regularized $\left(C_{1}, C_{2}\right)$-existence and uniqueness families, CUBO 17 (2015), 15-41.

[29] Fedorov, V. and Kostić, M., On a class of abstract degenerate multi-term fractional differential equations in locally convex spaces, preprint.

[30] Martinez, C. and Sanz, M., The Theory of Fractional Powers of Operators, North-Holland Math. Stud. 187, Elseiver, Amsterdam, 2001.

[31] Martinez, C., Sanz, M., and Redondo, A., Fractional powers of almost non-negative operators, Fract. Calc. Appl. Anal. 8 (2005), 201-230.

[32] Meise, R. and Vogt, D., Introduction to Functional Analysis, Translated from the German by M. S. Ramanujan and revised by the authors. Oxf. Grad. Texts Math., Clarendon Press, New York, 1997.

[33] Melnikova, I. V. and Filinkov, A. I., Abstract Cauchy Problems: Three Approaches, Chapman and Hall/CRC, Boca Raton, 2001.

[34] Melnikova, I. V., The Cauchy problem for differential inclusion in Banach space and distribution spaces, Siberian Math. J. 42 (2001), 751-765.

[35] Obukhovskii, V. and Zecca, P., On boundary value problems for degenerate differential inclusions in Banach spaces, Abstr. Appl. Anal. 13 (2003), 769-784.

[36] Podlubny, I., Fractional Differential Equations, Academic Press, New York, 1999.

[37] Prüss, J., Evolutionary Integral Equations and Applications, Birkhäuser-Verlag, Basel, 1993.

[38] Samko, S. G., Kilbas, A. A., and Marichev, O. I., Fractional Derivatives and Integrals: Theory and Applications, Gordon and Breach, New York, 1993.

[39] Straub, B., Fractional powers of operators with polynomially bounded resolvent and the semigroups generated by them, Hiroshima Math. J. 24 (1994), 529-548. 
[40] Sviridyuk, G. A. and Fedorov, V. E., Linear Sobolev Type Equations and Degenerate Semigroups of Operators, Inverse and Ill-Posed Problems (Book 42), VSP, Utrecht, Boston, 2003.

[41] Sviridyuk, G. A. and Vakarina, O. V., The Cauchy problem for a class of higher-order linear equations of Sobolev type, Differ. Uravn. 33 (1998), 1415-1424.

[42] Xiao, T.-J. and Liang, J., The Cauchy Problem for Higher-Order Abstract Differential Equations, Springer-Verlag, Berlin, 1998.

[43] Xiao, T.-J. and Liang, J., Abstract degenerate Cauchy problems in locally convex spaces, J. Math. Anal. Appl. 259 (2001), 398-412.

[44] Xiao, T.-J. and Liang, J., Higher order degenerate Cauchy problems in locally convex spaces, Math. Comp. Modelling 41 (2005), 837-847.

[45] Yagi, A., Generation theorem of semigroup for multivalued linear operators, Osaka J. Math. 28 (1991), 385-410.

[46] Zheng, Q. and Li, M., Regularized Semigroups and Non-Elliptic Differential Operators, Science Press, Beijing, 2014.

Faculty of Technical Sciences

University of Novi Sad

Trg D. Obradovića 6, 21125 Novi Sad, Serbia

E-mail: marco.s@verat.net 